UNIVERSIDADE DE SÃO PAULO

FACULDADE DE MEDICINA DE RIBEIRÃO PRETO

AMANDA PIRES BARBOSA

Correlação entre parâmetros clínicos e de Ressonância Nuclear Magnética da Síndrome de Sjögren e de pacientes com olho seco nãoSjögren 


\title{
Correlação entre parâmetros clínicos e de Ressonância Nuclear Magnética da Síndrome de Sjögren e de pacientes com olho seco não- Sjögren
}

\author{
Versão Corrigida \\ Versão original encontra-se na unidade que aloja o Programa de Pós- \\ Graduação
}

Tese apresentada à Faculdade de Medicina de Ribeirão Preto da Universidade de São Paulo para obtenção do Título de Doutor em Ciências.

Área de Concentração: Mecanismos Fisiopatológicos dos Sistemas Visual e ÁudioVestibular.

Orientador: Prof. Dr. Eduardo Melani Rocha 
AUTORIZO A REPRODUÇÃO E DIVULGAÇÃO TOTAL OU PARCIAL DESTE TRABALHO, POR QUALQUER MEIO CONVENCIONAL OU ELETRÔNICO, PARA FINS DE ESTUDO E PESQUISA, DESDE QUE CITADA A FONTE.

FICHA CATALOGRÁFICA

Barbosa, Amanda Pires.

Correlação entre parâmetros clínicos e de Ressonância Nuclear Magnética da Síndrome de Sjögren e de pacientes com olho seco nãoSjögren. / Amanda Pires Barbosa; Orientador, Eduardo Melani Rocha. Ribeirão Preto. - 2020.

77p.: 3il.; $30 \mathrm{~cm}$

Tese (Doutorado) - Programa de Oftalmologia, Otorrinolaringologia e Cirurgia de Cabeça e Pescoço. Área de Concentração: Mecanismos Fisiopatológicos dos Sistemas Visual e Áudio-Vestibular. Faculdade de Medicina de Ribeirão Preto da Universidade de São Paulo, Ribeirão Preto, 2020.

1. Olho seco. 2. Síndrome de Sjögren. 3. Glândula lacrimal. 4. Ressonância Nuclear Magnética. 


\section{FOLHA DE APROVAÇÃO}

Aluno: Amanda Pires Barbosa

Título: Correlação entre parâmetros clínicos e de Ressonância Nuclear Magnética da Síndrome de Sjögren e de pacientes com olho seco não-Sjögren.

Tese apresentada à Faculdade de Medicina de Ribeirão Preto da Universidade de São Paulo para obtenção do Título de Doutor em Ciências.

Área de Concentração: Mecanismos Fisiopatológicos dos Sistemas Visual e ÁudioVestibular.

Aprovado em:

Prof. Dr.

Instituição:

Assinatura:

Prof. Dr.

Instituição:

Assinatura:

Prof. Dr.

Instituição:

Assinatura:

Prof. Dr.

Instituição:

Assinatura: 


\section{Dedicatória}

Dedico minha Tese de Doutorado ao meu marido, João Paulo, e à minha filha, Helena. 


\section{Agradecimentos}

Agradeço primeiramente ao meu marido, aos meus pais, e à minha irmã Aline, que sempre estão próximos quando preciso, que me apoiam e me guiam a cada novo passo, que me aconselham e me fazem permanecer no caminho certo. Esta conquista é nossa.

Ao meu orientador e amigo, Prof. Dr. Eduardo Melani Rocha, por ser sempre muito solícito para esclarecer dúvidas e pelas várias sugestões que abrilhantaram 0 projeto. $O$ senhor foi fundamental na concretização deste sonho.

Ao meu coorientador, Dr. Denny Marcos Garcia, que me auxiliou na execução deste estudo.

Ao Departamento de Oftalmologia, Otorrinolaringologia e Cirurgia de Cabeça e Pescoço e Departamento de Radiologia e Diagnóstico por Imagem da Faculdade de Medicina de Ribeirão Preto da Universidade de São Paulo, em especial ao Prof Dr. Valdair Francisco Muglia, pelo apoio científico, e à CAPES, FAPESP e FAEPA, pelo auxílio financeiro.

À amiga Maria Cecília Onofre, pelo apoio técnico e ao Prof. Dr. José Carlos Barbosa (meu pai) pelas análises estatísticas.

Agradeço também às assistentes de pesquisa: Adriana de Andrade Batista Murashima, Carolina Maria Módulo e Marina Zilio Fantucci. Sem elas, tudo teria sido muito mais difícil.

Enfim, agradeço aos pacientes, que generosamente concordaram em participar desta pesquisa. Desejo que Deus os retribua com muita saúde e paz! 


\section{Apoio Financeiro}

Agradeço à CAPES (Coordenação de Aperfeiçoamento de Pessoal de Nível Superior), pela concessão da bolsa. 
Barbosa AP. Correlação entre parâmetros clínicos e de Ressonância Nuclear Magnética da Síndrome de Sjögren e de pacientes com olho seco não-Sjögren. Tese (Doutorado) - Faculdade de Medicina de Ribeirão Preto, Universidade de São Paulo. Ribeirão Preto. 2020.

Introdução: A doença do olho seco (DOS) é uma desordem frequente do filme lacrimal, cujos sintomas podem indicar a manifestação de uma condição sistêmica, por exemplo, a Síndrome de Sjögren (SS). No entanto, o diagnóstico da DOS representa um desafio, e tem se observado a utilização crescente de exames de imagem para avaliação de glândulas lacrimais $(G L)$, incluindo as de pacientes com SS. Objetivo: Avaliar se o olho seco está associado a alterações morfológicas da GL analisada em imagens de Ressonância Nuclear Magnética (RNM), comparandose doentes portadores de SS e pacientes com olho seco não-SS. Casuística e Métodos: Foram convidados 27 pacientes com SS e 11 com DOS não-SS para obtenção de teste de Schirmer I, coloração corneana por fluoresceína, tempo de ruptura do filme lacrimal, fluxo salivar e questionário sobre índice da doença da superfície ocular (OSDI). Aqueles que não possuíam RNM concordaram em realizar o exame. As imagens da RNM de todos os pacientes e de 29 controles foram avaliadas quanto a: volume da GL; intensidade de sinal da GL pela do vítreo (GL/V); intensidade de sinal da glândula parótida pela do vítreo (GP/V); coeficiente Aparente de Difusão (ADC) da GL; ADC da GP e volume do gânglio trigêmeo (GT). Os resultados foram comparados entre os três grupos e em diferentes faixas etárias. Posteriormente, os resultados radiológicos foram comparados com os dados clínicos dos doentes. Resultados: Observou-se tendência à redução da média do volume da GL com aumento da idade em todos os grupos, e os pacientes idosos com SS apresentaram os menores valores, embora sem significância estatística. A média do volume do GT foi maior nos pacientes com olho seco não-SS, em relação ao grupo com SS $(p=0,02)$. O grupo controle demonstrou valores de ADC maiores que os dos outros grupos, embora sem significância estatística. No grupo com SS, a presença de ceratite puntata apresentou correlação positiva $(p=0,03)$ com intensidade de sinal da GP/V e negativa ( $p=0,0002)$ com o ADC da $G L ; 0$ volume da GL apresentou médias maiores nos pacientes com maior fluxo salivar $(p=0,01)$, e os doentes com baixa produção de lágrima evidenciaram maior intensidade de sinal $\operatorname{GP} / V(p=0,02)$. Conclusões: A RNM é capaz de monitorar alterações da GL e, no grupo com SS, os resultados radiológicos se correlacionaram aos dados clínicos.

Palavras-chave: Olho seco. Síndrome de Sjögren. Glândula lacrimal. Ressonância Nuclear Magnética. 
Abstract 
Barbosa AP. Correlation between clinical and magnetic nuclear resonance parameters of Sjögren's syndrome and patients with non-Sjögren dry eye. Tese (Doutorado) - Faculdade de Medicina de Ribeirão Preto, Universidade de São Paulo. Ribeirão Preto. 2020.

Introduction: Dry eye disease (DED) is a frequent tear film disorder whose symptoms may signify the manifestation of a systemic condition, for example, Sjögren's Syndrome (SS). However, the diagnosis of DED is challenging, and there has been a growing use of imaging tests to assess the lacrimal glands (LG), including those of patients with SS. Aims: To evaluate whether dry eye is associated with morphological changes in LG analyzed in MRI images, comparing SS patients and patients with non-SS dry eye. Casuistic and Methods: Twenty-seven patients with SS and 11 with non-SS DED were invited to obtain the Schirmer test I, corneal fluorescein staining, tear breakup time (TBUT), salivary flow and ocular surface disease index (OSDI) questionnaire; those who did not have MRI agreed to perform the exam. MRI images of all patients and 29 controls were assessed for: LG volume; LG-to-vitreous body signal ratio (LG/V); Parotid gland-to-vitreous body signal ratio (PG/V); apparent diffusion coefficient (ADC) of LG; $A D C$ of $P G$ and trigeminal ganglion volume (TG). Results were compared between the 3 groups and in different age groups. Subsequently, the radiological parameters were compared with the clinical data of the patients. Results: There was a tendency to reduce the mean volume of LG with increasing age in all groups, and elderly patients with SS presented the lowest values, although there was no statistical significance. The mean TG volume was higher in non-SS dry eye patients compared to SS group ( $p=0.02$ ). The control group showed higher ADC values than the other groups, although without statistical significance. In the SS group, the presence of keratitis puntata was positively correlated $(p=0.03)$ with $P G / V$ signal intensity and negative $(p=0.0002)$ with LG ADC; LG volume presented higher averages in patients with higher salivary flow $(p=0.01)$, and patients with low tear production presented higher PG/V signal intensity $(p=0.02)$. Conclusions: MRI is able to monitor changes in LG in SS, and the radiologic data correlated with clinical data in SS group.

Keywords: Dry eye. Sjögren's Syndrome. Nuclear Magnetic Resonance. Lacrimal gland. 
Lista de Figuras 
Figura 1 - Deslocamento das moléculas de água entre os espaços intra e extracelular e dentro do espaço extracelular (setas). Em A a movimentação das moléculas de água é restrita devido à maior quantidade de células existentes; em B a celularidade é menor, com consequente maior movimentação das moléculas

Figura 2 - Imagem de glândual lacrimal normal em corte axial em T1 (setas brancas)......

Figura 2 - Imagem de GP normal em corte axial em T2, com alta intensidade de sinal (asterisco). A assimetria entre a GP direita e a esquerda se deve à rotação no plano transversal

Figura 3 - Imagem axial em T1 mostrando a porção cisternal do nervo trigeminal (setas) e o cavum de Meckel (asterisco) 
Tabela 1 - Média do volume da glândula lacrimal do grupo controle .50

Tabela 2 - Média da razão intensidade sinal glândula lacrimal/vítreo do grupo controle

Tabela 3 - Média do coeficiente aparente de difusão da glândula lacrimal do grupo controle.

Tabela 4 - Comparação entre as médias dos parâmetros da ressonância nuclear magnética.

Tabela 5 - Média do volume da glândula lacrimal $\left(\mathrm{cm}^{3}\right)$ à ressonância nuclear magnética por faixa etária

Tabela 6 - Média da razão intensidade sinal glândula lacrimal/vítreo à ressonância nuclear magnética por faixa etária

Tabela 7 - Média da razão intensidade sinal glândula parótida/vítreo à ressonância nuclear magnética por faixa etária

Tabela 8 - Média do coeficiente de difusão da glândula lacrimal à ressonância nucelar magnética por faixa etária

Tabela 9 - Média do coeficiente de difusão da glândula parótida à ressonância nuclear magnética por faixa etária

Tabela 10 - Média do volume do gânglio trigêmeo $\left(\mathrm{mm}^{3}\right)$ à ressonância nuclear magnética por faixa etária....

Tabela 11 - Correlação entre as médias dos parâmetros da ressonância nuclear magnética de pacientes com Síndrome de Sjögren e dados clínicos utilizando o coeficiente de correlação Pearson

Tabela 12 - Correlação entre as médias dos parâmetros da ressonância nuclear magnética e dados clínicos de pacientes com olho seco não-Síndrome de Sjögren utilizando o coeficiente de correlação Pearson.

Tabela 13 - Dados clínicos dos pacientes do grupo Síndrome de Sjögren

Tabela 14 - Dados clínicos dos pacientes do grupo Não-Síndrome de Sjögren....82 
Lista de Símbolos e Abreviaturas 
ADC- $\quad$ Coeficiente aparente de difusão

AIDS- Síndrome da imunodeficiência adquirida

anti-MUC3- Anticorpos contra receptores muscarínicos

$\mathbf{c m}^{3}-\quad$ centímetros cúbicos

DICOM- $\quad$ Digital Imaging and Communication in Medicine

DOS- Doença do olho seco

EUA- $\quad$ Estados Unidos da América

FAN- $\quad$ Fator antinúcleo

FR- $\quad$ Fator reumatoide

GL- $\quad$ Glândulas lacrimais

GP- $\quad$ Glândula parótida

GS- Glândulas salivares

GT- Gânglio trigêmeo

HCFMRP-USP- Hospital das Clínicas da Faculdade de Medicina de Ribeirão Preto da Universidade de São Paulo

HIV- $\quad$ Vírus da Imunodeficiência Humana

OD- Olho direito

OE- $\quad$ Olho esquerdo

OSDI- Índice da doença da superfície ocular

RF- $\quad$ Radiofrequência

RNM- $\quad$ Ressonância Nuclear Magnética

SS- $\quad$ Síndrome de Sjögren

SS-A- anti-Ro

SS-B- anti-La

STIR- $\quad$ Sequência de recuperação de inversão de tempo curto 
TC-

Tomografia Computadorizada

TFBUT-

Tempo de ruptura do filme lacrimal (do inglês Tear Film Breakup Time)

TRFL-

Tempo de ruptura do filme lacrimal

US-

Ultrassonografia

V-

Vítreo 


\section{SUMÁRIO}

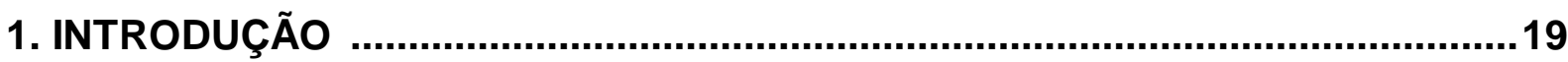

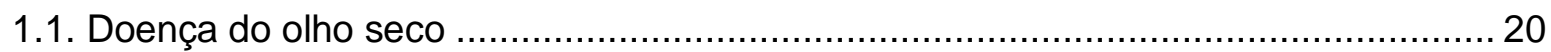

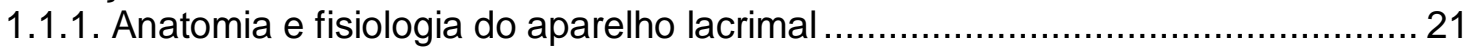

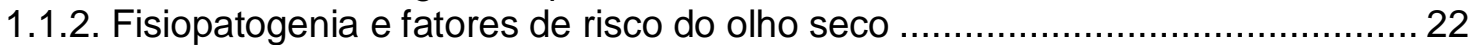

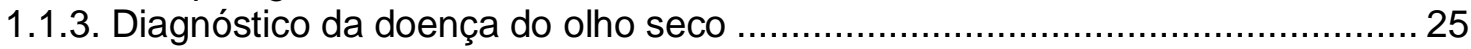

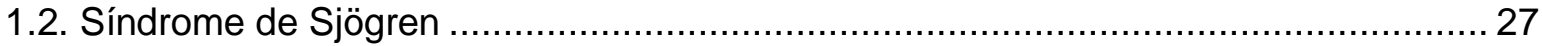

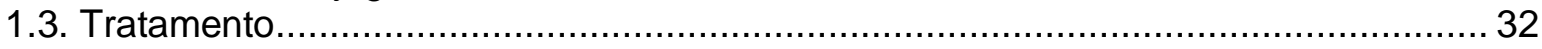

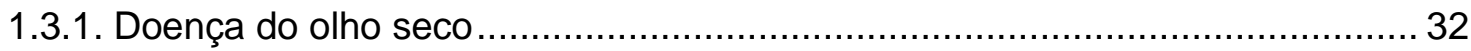

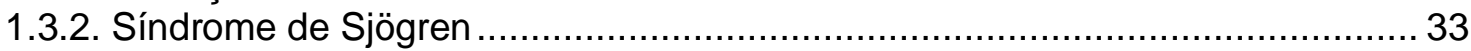

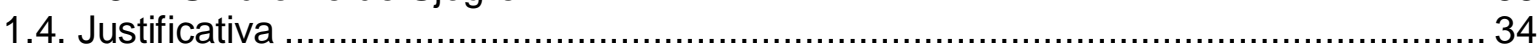

1.5. Exames de imagem para avaliação de doenças das glândulas lacrimal e parótida ... 35

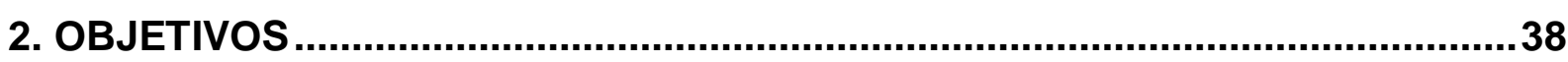

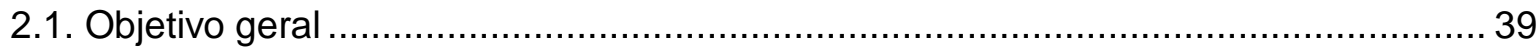

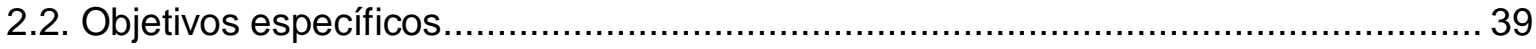

3. CASUÍSTICA E MÉTODOS ............................................................................. 40

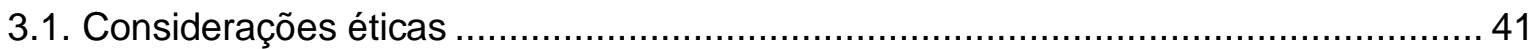

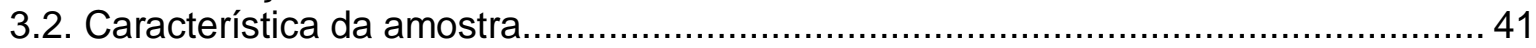

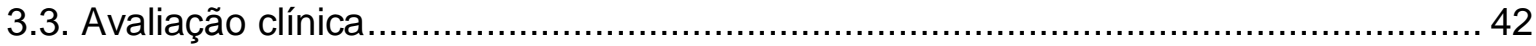

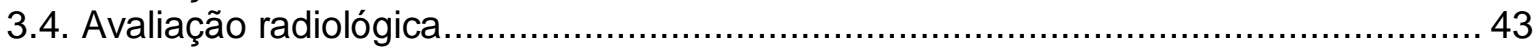

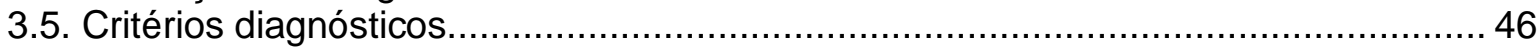

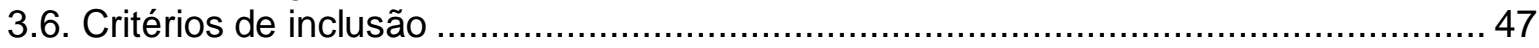

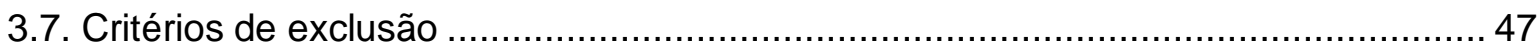

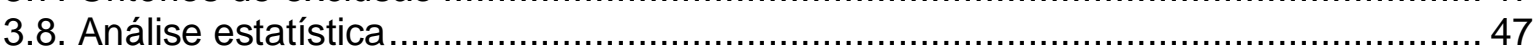

3.9. Análise crítica dos riscos e benefícios ............................................................. 48

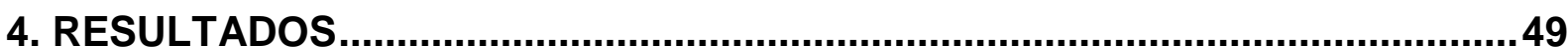

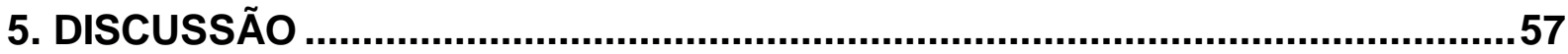

6. CONCLUSÕES .......................................................................................... 62

7. REFERÊNCIAS BIBLIOGRÁFICAS .......................................................64

8. ANEXOS 
1- Introdução 
A doença do olho seco (DOS) é uma desordem do filme lacrimal causada por diminuição da produção ou excesso de evaporação da lágrima. Caracteriza-se por inflamação da superfície ocular, associa-se a queixas de desconforto e seus sintomas podem significar a manifestação de uma condição sistêmica, por exemplo, a Síndrome de Sjögren (Lemp, 1995). No entanto, o diagnóstico da DOS representa um desafio, uma vez que diversos autores têm definido diferentes critérios para este fim, e não há consenso em relação ao assunto (Javadi; Feizi, 2011). Soma-se a isto o fato de os sinais e sintomas nem sempre se correlacionarem nos pacientes (Savini et al., 2008).

A utilização crescente de exames de imagem tem auxiliado no diagnóstico e seguimento de doenças das glândulas lacrimais (GL), de modo que alguns autores sugerem sua realização antes de procedimentos invasivos, em situações suspeitas de alterações nesses tecidos (Sahinoglu et al., 2011).

Pelo fato de a DOS se tratar de uma condição cada vez mais frequente, multifatorial, com apresentação clínica variada e discordância entre os sinais e sintomas nela presentes, este estudo foi realizado com o objetivo de avaliar se 0 olho seco está associado a alterações morfológicas da GL analisada em imagens de Ressonância Nuclear Magnética (RNM).

\subsection{Doença do olho seco}

Olho seco é definido como doença multifatorial da superfície ocular, caracterizada por instabilidade e hiperosmolaridade do filme lacrimal, inflamação e anormalidades neurossensoriais, sendo acompanhada de sintomas oculares (Craig et al., 2017). As queixas mais comuns são sensação de corpo estranho, queimação, prurido, fotofobia, e embaçamento visual. Pacientes com quadro grave podem apresentar úlcera e consequente perfuração da córnea (Fonseca; Arruda; Rocha, 2010). Provoca redução da qualidade de vida devido à dor ocular significativa, limitação da realização de atividades diárias como leitura e até depressão (Gonzales et al., 2018). A prevalência mundial da doença varia de $5 \%$ a $50 \%$ da população, mas pode ser maior do que $75 \%$ em adultos acima de 40 anos, sendo as mulheres mais acometidas (Stapleton et al., 2017). Nos Estados Unidos da América (EUA) 
estima-se que $7 \%$ das mulheres e $4 \%$ dos homens acima de 50 anos apresentem sintomas de ressecamento ocular (Schaumberg et al., 2003). Estudos realizados mostraram taxa de 25\% de olho seco no Canadá (Doughty et al., 1997), 33\% no Japão (Shimmura; Shimazaki; Tsubota, 1999) e 7,4\% na Austrália (McCarty et al., 1998), enquanto na Indonésia (Lee et al., 2002) e em Taiwan (Lin et al., 2003), esse número é de $27,5 \%$ e $33,7 \%$, respectivamente. No Brasil, a prevalência encontrada foi de 12,8\% (Castro et al., 2018).

Trata-se de uma das mais frequentes morbidades oculares, de modo que em torno de $25 \%$ dos pacientes que procuram clínicas oftalmológicas relatam sintomas de olho seco, tornando-a um crescente problema público de saúde. O impacto econômico anual gerado pelo manejo da morbidade pode alcançar, em média, \$783 por paciente nos EUA, sendo o custo total à economia do país em torno de $\$ 3.8$ bilhões. Considerando-se o prejuízo às atividades laborais, os custos à sociedade são estimados em $\$ 11,302$ por paciente e $\$ 55.4$ bilhões àquela nação (Yu; Asche; Fairchild, 2011).

\subsubsection{Anatomia e fisiologia do aparelho lacrimal}

As GL principais e acessórias excretam a lágrima, que desce por efeito da gravidade em direção à pálpebra inferior, penetra nos pontos lacrimais, de onde alcança os canalículos, em seguida atinge o saco lacrimal e, por último, o ducto nasolacrimal, chegando ao meato nasal inferior (Volpini et al., 2013).

A GL principal é classificada como uma glândula exócrina mucosa e serosa, portanto mista, está localizada na fossa lacrimal do frontal, no terço lateral do teto orbitário, à frente da gordura orbitária e atrás do septo orbitário. Apresenta cor amarelo-avermelhada, forma bilobada e as seguintes dimensões: $20 \mathrm{~mm}$ de comprimento, $15 \mathrm{~mm}$ de largura, 3 a $5 \mathrm{~mm}$ de espessura. $O$ seu peso é em torno de 60 a 80 gramas. A asa lateral do músculo levantador da pálpebra superior a divide em porção orbitária (posterior e superior) e porção palpebral (anterior e inferior) (Dantas, 2013).

O filme lacrimal é formado por três camadas: a mais externa é a lipídica, que consiste em ésteres alifáticos e colesterol, e previne a evaporação da lágrima, sendo 
produzida por glândulas localizadas na margem ciliar, chamadas de Meibomius, Moll e Zeis. A camada intermediária é a aquosa, mais volumosa, contém cloreto de sódio, potássio, glicose, albumina, lisozima, globulina e imunoglobulinas, e é secretada pela GL principal e por glândulas lacrimais acessórias localizadas na conjuntiva palpebral, chamadas de Krause e Wolfring. Esta porção serosa é reponsável por proteger a superfície ocular contra agentes infecciosos, controlar a osmolalidade do filme lacrimal e o $\mathrm{pH}$, além de permitir que a lágrima se espalhe mais facilmente e lubrifique o olho. A camada mais interna é a mucoide, formada por uma glicoproteína que apresenta ácido siálico e hexosaminas; é secretada pelas glândulas de Henle, presentes na conjuntiva tarsal, e pelas células caliciformes da prega semilunar e carúncula. Sua função é umidificar e auxiliar a margem palpebral e a conjuntiva a deslizarem suavemente durante o piscar e os movimentos oculares, além de proteger a córnea e a conjuntiva de abrasões provenientes de corpo estranho (Dantas, 2013; Masmali; Purslow; Murphy, 2014).

A secreção lacrimal é regulada pela resposta neural derivada dos nervos aferentes sensoriais da superfície ocular, através do nervo lacrimal, ramo do nervo oftálmico, que por sua vez é ramo do trigêmeo. Estímulos aferentes sensoriais na córnea e conjuntiva ativam fibras eferentes simpáticas e parassimpáticas, que em seguida conduzem impulsos para a GL e glândulas acessórias, iniciando a secreção e excreção da lágrima (Dartt, 2009). As fibras aferentes da GL representam a maior parte das fibras constituintes do trigêmeo, cujas células localizam-se no gânglio trigeminal (Dantas, 2013). As GL, a superfície ocular (córnea, conjuntiva e glândulas meibomianas), as pálpebras e os nervos sensoriais e motores que as integram constituem um sistema integrado chamado Unidade Funcional Lacrimal, cuja disfunção provoca alterações patológicas na composição da lágrima (Stern et al., 2004).

\subsubsection{Fisiopatogenia e fatores de risco do olho seco}

A DOS apresenta como principal característica a hiperosmolaridade do filme lacrimal, o que promove dano à superfície ocular direta e indiretamente, por meio da indução de processo inflamatório. Em indivíduos susceptíveis, um estímulo 
ambiental pode iniciar a agressão aos tecidos envolvidos, gerando respostas neuropáticas, metabólicas e/ou inflamatórias. Estas, por sua vez, levam à instabilidade do filme lacrimal, diminuição da secreção lacrimal, ao aumento da evaporação ou à alteração da composição da lágrima, o que leva à perpetuação do processo inflamatório, constituindo um ciclo vicioso (Fonseca; Arruda; Rocha, 2010).

A doença é classificada etiologicamente em duas categorias que podem coexistir: olho seco por deficiência aquosa e olho seco evaporativo. A primeira envolve as condições que afetam a função da GL, reduzindo a secreção da lágrima; a última se relaciona com a evaporação excessiva da lágrima por sua exposição, e inclui tanto as causas relacionadas à disfunção das pálpebras e do piscar, quanto as disfunções da superfície ocular (Craig et al., 2017).

A deficiência aquosa subdivide-se em: Síndrome de Sjögren, primária ou secundária; e não associada à síndrome de Sjögren, (Lemp, 1995). Esta última, por sua vez, compreende as seguintes causas:

- Olho seco relacionado à idade: a apoptose celular que ocorre com o envelhecimento afeta todas as glândulas exócrinas, com consequente ressecamento generalizado. A secreção lacrimal começa a reduzir a partir dos 30 anos, e por volta dos 60 anos se torna insufficiente para as necessidades basais (Murube et al., 2005).

- Alacrimia congênita: causa rara de olho seco em jovens, determinada por mutação genética, ocorre na síndrome do triplo A (síndrome de Allgrove), doença de Addison, neurodegeneração central e disfunção autonômica (Davidoff; Firedman, 1977).

- Condições sistêmicas como sarcoidose, hemocromatose, linfoma, amiloidose e infecções virais (Epstein-Barr, Vírus da Imunodeficiência Humana - HIV, Vírus Linfotrópico da célula T humana 1) resultam em infiltração inflamatória da GL e substituição dos ácinos secretórios (American Academy of Ophthalmology, 2013). 
- Transplante de células tronco hematopoiéticas (principalmente alogênico), com ou sem a ocorrência da doença do enxerto-hospedeiro (Ivanir et al., 2013).

- Obstrução dos ductos da GL por tracoma, penfigoide cicatricial e de membrana mucosa, eritema multiforme, queimaduras químicas ou térmicas e após terapia radioativa (Holland; Mannis; Lee, 1998).

- Redução do estímulo sensorial a partir da superfície ocular com consequente hiposecreção reflexo-induzida, o que ocorre em casos de uso crônico de lentes de contato, levando à hipoestesia da córnea, e na diabetes mellitus, em associação à neuropatia autonômica ou sensorial (Fonseca; Arruda; Rocha, 2010).

- Cirurgia refrativa a laser, devido à secção de nervos corneanos, induzindo à diminuição transitória da sensibilidade corneal, resultando em menor produção do conteúdo aquoso da lágrima, por diminuição do estímulo eferente (Credie et al., 2007).

Os fatores relacionados ao olho seco evaporativo englobam:

- conjuntivite alérgica, uso de colírios contendo conservantes e lentes de contato promovem quebra precoce do filme lacrimal, com subsequente hipermosmolaridade da lágrima. A disfunção da glândula de Meibomius é outra condição que leva ao aumento da osmolaridade devido à deficiência da camada lipídica do filme lacrimal (Bron et al., 2017).

- Mau posicionamento ou disfunção palpebral: as pálpebras também apresentam relevância na distribuição, no armazenamento e na drenagem das lágrimas, de modo que essas desordens podem provocar ressecamento devido exposição ocular prolongada (Dantas, 2013).

- Medicações como anti-histamínicos e antidepressivos (Terry et al., 2001).

- Hábitos como tabagismo, baixa ingesta de ômega 3, uso de computador por longos períodos, leitura prolongada (Gayton, 2009).

- Mulheres na menopausa e pós-menopausa, uma vez que a deficiência de androgênios predispõe à disfunção da GL e das glândulas de Meibomius, o que aumenta o risco de instabilidade do filme lacrimal (Sullivan et al., 
2017). Terapia de reposição hormonal no período pós-menopausa aumenta a prevalência de DOS, principalmente em mulheres que utilizam apenas estrogênio (Schaumberg et al., 2001).

- Deficiência de vitamina A causa ceratinização da superfície ocular e redução das células caliciformes (Tei et al., 2000).

A patogênese do olho seco também envolve a ocorrência de estresse oxidativo, com liberação de radicais livres e espécies reativas ao oxigênio que podem gerar apoptose e necrose celular. Este mecanismo está presente em diversas condições sistêmicas, como doenças neurodegenerativas, cardiovasculares, câncer e doenças oculares como degeneração macular relacionada à idade, catarata, uveíte, retinopatia da prematuridade e alterações corneanas. Os radicais livres promovem lesão celular resultante da peroxidação lipídica das membranas, modificação oxidativa de proteínas e dano oxidativo ao DNA (Wakamatsu; Dogru; Tsubota, 2008). A hiperglicemia em situações de diabetes mellitus e resistência à insulina também provocou estresse oxidativo e, consequentemente, alterações histológicas na GL de ratos, caracterizadas por mudanças na morfologia e acúmulo de inclusões semelhantes à lipofuscina (Módulo et al., 2009).

A deficiência aquosa gerada pela Síndrome de Sjögren será detalhada em tópico específico.

\subsubsection{Diagnóstico da doença do olho seco}

Diversos questionários, formalmente aceitos, estão disponíveis para auxiliar na detecção de casos de olho seco subclínicos e na avaliação do impacto da doença na qualidade de vida (Zeev; Miller; Latkany, 2014). Além desta ferramenta, existem diversos testes para analisar a estabilidade do filme lacrimal, a superfície ocular e a produção/evaporação de lágrima, a saber:

- Coloração da superfície ocular com fluoresceína sódica $2 \%$, rosa bengala $1 \%$ e lisamina verde $1 \%$. A fluoresceína cora as regiões em que as células epiteliais corneanas e conjuntivais estejam ausentes, ou cuja 
membrana esteja danificada, e é estimulada pela luz azul cobalto, utilizada na lâmpada de fenda (Begley et al., 2019). O rosa bengala é um corante vital derivado da fluoresceína sódica, corando em rosa células degeneradas e mortas da superfície ocular, observadas sob luz branca à biomicroscopia; no entanto, sua instilação provoca ardor, sendo pouco tolerado (Argüeso et al., 2006). A lisamina verde cora células epiteliais que tiveram suas membranas danificadas, necessita de filtro vermelho para melhorar sua visualização e é bem tolerada (Wolffsohn et al., 2017).

- Tempo de ruptura do filme lacrimal (Tear Film Breakup Time - TFBUT): após a instilação de fluoresceína, a função lacrimal é avaliada medindo quantos segundos a lágrima demora para se romper na superfície ocular após o piscar. À biomicroscopia, o ressecamento corneano é mostrado como áreas escuras, sendo que um TFBUT menor que 10 segundos é considerado patológico (Dohlman; Ciralsky; Lai, 2016).

- Altura ou espessura do menisco lacrimal, observada durante exame à lâmpada de fenda; pode ser medida de forma objetiva utilizando-se tomografia de coerência óptica. Valores abaixo de $0.2 \mathrm{~mm}$ são sugestivos de hipossecreção lacrimal (Messmer, 2015).

- Teste de Schirmer: mede a produção aquosa da lágrima, por meio de fitas de papel colocadas no saco conjuntival inferior, sendo permitido ao paciente piscar. A fita apresenta marcação milimetrada que absorve a lágrima produzida durante 5 minutos, de modo que medição menor do que $10 \mathrm{~mm}$ é indicativa de olho seco. Há três formas de realizar o exame: (a) sem anestesia tópica (Teste de Schirmer I), que avalia a habilidade da superfície ocular de responder a um estímulo e, portanto, a secreção basal e reflexa; (b) sob anestesia tópica (Teste de Schirmer-Jones), que avalia a secreção lacrimal basal e (c) Teste de Schirmer II, em que é provocada a estimulação da mucosa nasal com a extremidade de um filamento de algodão, para se obter o máximo de lacrimejamento reflexo. Entretanto, o exame está sujeito a alterações ambientais e psicológicas, podendo variar os resultados (Dantas, 1999; Javadi; Feizi, 2011). 
- Avaliação da morfologia e do padrão de secreção das glândulas de Meibômio: nos estágios iniciais da disfunção dessas glândulas é possível utilizar um método não invasivo, a Meibografia com luz infravermelha, para demonstrar anormalidades como irregularidade, dilatação, distorção e obstrução glandulares (Arita; Fukuoka; Morishige, 2017; LlorensQuintana et al., 2019). Em casos mais avançados, é possível visualizar tais alterações apenas por meio da biomicroscopia com lâmpada de fenda (Geerling et al., 2017). A qualidade e a quantidade de secreção produzida são avaliadas, na prática clínica, por meio da expressão digital do tarso, sendo visualizada substância oleosa clara em situações normais, enquanto que na disfunção glandular é observada secreção semelhante à pasta de dente (Tomlinson et al., 2011).

Os valores de referência estabelecidos para esses exames, no entanto, são variáveis, sendo que a plataforma mais aceita atualmente é a apresentada pela Sociedade de Superfície Ocular e Filme Lacrimal (Tear Film \& Ocular Surface Society - Dry Eye Workshop II) (Craig et al., 2017).

\subsection{Síndrome de Sjögren}

A SS é uma doença crônica inflamatória autoimune que afeta caracteristicamente as GL e as glândulas salivares (GS), ocasionando sintomas de olho seco e boca seca (Fox, 2005), devido infiltração linfocítica focal dessas glândulas (Moutsopoulos, 1994). A doença pode também envolver numerosos outros órgãos, como pulmões, rins, articulações, nervos periféricos e o cérebro (Felberg; Dantas, 2006). Pacientes com SS apresentam elevada incidência de linfoma maligno de células B tipo não-Hodgkin (Asmussen et al., 1997). Na maioria dos casos, a síndrome ocorre isoladamente, sendo chamada de SS primária. Entretanto, pode haver associação com outra doença inflamatória ou automimune, principalmente artrite reumatoide, doença tireoidiana autoimune, escledrodermia e lupus eritematoso sistêmico; neste caso, é designada SS secundária (Asmussen; Bowman, 2001). 
Em relação à epidemiologia, as mulheres são mais acometidas do que os homens, com relação de 9:1 a 19:1. A média de idade ao diagnóstico para SS primária é de 56 anos (Stefanski et al., 2017). Recente metanálise realizada com 18 estudos computou prevalência de $0,06 \%$ no mundo (Qin et al., 2015). Quanto à etiologia, infecções virais prévias (vírus Epstein-Barr, citomegalovírus, herpes vírus humano, vírus da hepatite $\mathrm{C}$ dentre outros) ou bacterianas (Helicobacter pylori) foram descritas como potenciais desencadeadores da resposta imune ao tecido glandular, devido a frequente concomitância em pacientes com SS (Abe et al., 1999; Aragona et al., 1999). Devido ao maior acometimento de mulheres, disfunções hormonais parecem fazer parte da fisiopatologia no desenvolvimento da SS, principalmente as deficiências de andrógenos, estrógeno e progesterona (Hayashi; Arakaki; Ishimaru, 2004); além disso, há evidências de um componente genético etiológico, em razão da observação de agregação de doenças autoimunes em familiares de pacientes com SS, possivelmente autossômico dominante (Kassan, 1998).

A patogênese da doença envolve hiper-reatividade dos linfócitos $B$, que se convertem em plasmócitos e produzem anticorpos contra antígenos do epitélio dos ácinos e dos ductos das glândulas exócrinas. São também ativados Linfócitos $T$ supressores, perpetuando a atividade dos linfócitos B ativados e a agressão tecidual (Yamamoto, 2003).

Estão presentes na doença anticorpos órgãos-específicos contra antígenos nos ductos glandulares, tireoide, mucosa gástrica, eritrócitos, pâncreas, próstata e células nervosas, e autoanticorpos não específicos, como fator reumatoide (FR), fator antinúcleo (FAN), anticorpo antimitocondrial, anticorpo anticentrômero, dentre outros. São frequentes nos pacientes com SS primária anticorpos contra ribonucleoproteínas: anti-Ro (SS-A) e anti-La (SS-B), enquanto o FR e FAN são mais frequentes na SS secundária. Autoanticorpos contra receptores muscarínicos (anti-MUC3) e contra proteínas do citoesqueleto das células acinares (anti-alfafodrim) também estão circulantes no sangue periférico (Tong L; Koh; Thong BY, 2017). 
A SS apresenta evolução clínica variável, amplo espectro de manifestações clínicas e muitos sintomas inespecíficos, o que dificulta seu diagnóstico precoce (Kruszka; O'Brian, 2009).

Os critérios mais aceitos para o estabelecimento do diagnóstico da SS são:

1) Critérios de San Francisco para o diagnóstico de SS primária e SS secundária (Daniels; Whitcher, 1994).

- SS primária

a) Biópsia de GS menor evidenciando sialoadenite focal com infiltração linfocitária com mais de um "focus" $/ 4 \mathrm{~mm}^{2}$ (Daniels et al., 2011), ou lesão benigna linfo-epitelial localizada em GS maior.

b) Diagnóstico de cérato-conjuntivite seca

- uso de Rosa Bengala demonstrando comprometimento córneoconjuntival;

- redução do menisco lacrimal ou do tempo de ruptura do filme lacrimal;

- teste de Schirmer I (sem anestésico) menor ou igual a $5 \mathrm{~mm}$ em 5 minutos.

-SS secundária

a) Presença de artrite reumatoide ou outra doença do tecido conectivo;

b) Pelo menos um dos itens descritos para o diagnóstico da SS primária. Possível SS: pelo menos um dos itens descritos para o diagnóstico da SS primária e uma das doenças a seguir: infiltrado linfocítico pulmonar, nefrite intersticial, púrpura, hepatite crônica (sem cirrose ou infecção), neuropatia periférica e hiper-gamaglobulinemia.

2) Critérios de San Diego para o diagnóstico de SS (Fox et al., 1986):

a) evidência objetiva de cérato-conjuntivite seca, documentada com coloração por Rosa Bengala ou fluoresceína;

b) evidência objetiva da diminuição do fluxo salivar; 
c) biópsia de GS menor, contendo pelo menos quatro lóbulos e com presença de pelo menos dois "foci"/4 $\mathrm{mm}^{2}$ (Daniels et al., 2011).

d) Presença de doença autoimune sistêmica comprovada por autoanticorpos séricos como FR, FAN, SS-A e SS-B.

- SS: quando os quatro itens são encontrados.

- Possível SS: quando três itens estão presentes.

- Exclusão: doenças existentes como linfoma, infecção pelo HIV, uso de medicações que sabidamente causam olho seco, sarcoidose, doença enxerto $\mathrm{x}$ hospedeiro.

3) Critérios europeus modificados pelo Grupo de Consenso AmericanoEuropeu (Vitali et al., 2002):

1. Sintomas oculares: pelo menos uma resposta afirmativa para uma das três questões formuladas abaixo:

a) Tem problemas oculares diários e persistentes, relacionados a quadro de olho seco há mais de três meses?

b) Tem sensação de areia ou queimação ocular?

c) Usa colírios lubrificantes mais de três vezes ao dia?

2. Sintomas orais: pelo menos uma resposta afirmativa para uma das três questões formuladas abaixo:

a) Tem sensação de boca seca há mais de três meses?

b) Tem inchaço recorrente ou persistente das GS, na idade adulta?

c) Sente necessidade de ingerir líquidos para ajudar na deglutição de alimentos sólidos?

3. Sinais oculares: Evidencia de modo objetivo o comprometimento ocular, quando pelo menos um dos dois testes abaixo é positivo:

a) Teste de Schirmer I ( $\leq 5 \mathrm{~mm}$ em 5 minutos)

b) Rosa Bengala $\geq 4$ pontos na escala de Bijsterveld (van Bijsterveld, 1969) 
4. Achados histopatológicos: Aglomeração de pelo menos 50 células mononucleares numa biópsia de $4 \mathrm{~mm}^{2}$ da GS (Daniels et al., 2011).

5. Comprometimento da GS: Evidencia de modo objetivo o comprometimento dessas glândulas, com pelo menos um dos três métodos abaixo.

a) Cintilografia da GS;

b) Sialografia da GP;

c) Fluxo salivar sem estímulo reflexo (Navazesh, 1993) $\leq 1,5 \mathrm{~mL}$ em 15 minutos.

6. Autoanticorpos: presença de pelo menos um dos seguintes autoanticorpos séricos:

a) Anticorpos contra os antígenos Ro/SS-A ou La/SS-B;

b) Anticorpos antinuclear;

c) Fator reumatoide.

Critérios de exclusão: Linfoma pré-existente, síndrome da imunodeficiência adquirida (AIDS), sarcoidose ou doença do enxerto $x$ hospedeiro.

- Provável SS primária: presença de pelo menos três dos seis itens.

- SS primária: presença de pelo menos quatro dos seis itens (aceitando como padrão sorológico positivo apenas SS-A ou SS-B).

- Provável SS secundária: combinação da resposta positiva para os itens 1 ou 2, com pelo menos um item positivos entre as questões 3,4 ou 5 .

- SS secundária: combinação da resposta positiva para os itens 1 ou 2, com pelo menos dois itens positivos entre as questões 3,4 ou 5 .

4. Critérios do Colégio Americano de Reumatologia/Liga Europeia contra Reumatismo (Shiboski et al., 2017).

Combinação de a) e b):

a) Pelo menos um sintoma ocular ou oral (resposta positiva a pelo menos uma das seguintes questões): 
- Apresenta desconforto devido olho seco diariamente e de forma persistente há mais de três meses?

- Tem sensação recorrente de areia nos olhos?

- Utiliza lágrimas artificiais mais do que três vezes por dia?

- Apresenta sensação de boca seca diariamente há mais de três meses?

- Necessita ingerir líquidos frequentemente para engolir alimentos secos?

b) Escore maior ou igual a quatro pontos:

- GS labial com sialodenite focal linfocítica e focus escore $\geq 1.3$ (Daniels et al., 2011) (três pontos)

- Anti-SSA (Ro) positivo (três pontos)

- Coloração da superfície ocular $\geq 5$ na escala de Whitcher (Whitcher et al., 2010) ou $\geq 4$ na escala de van Bijsterfeld (van Bijsterveld, 1969) em pelo menos um olho (um ponto)

- Schirmer $\leq 5 \mathrm{~mm} / 5 \mathrm{~min}$ em pelo menos um olho (um ponto)

- Fluxo salivar sem estímulo $\leq 0.1 \mathrm{ml} / \mathrm{min}$ (Navazesh, 1993) (um ponto)

Critérios de exclusão: tratamento radioativo prévio de cabeça e pescoço, hepatite C ativa (com PCR positivo), AIDS, sarcoidose, amiloidose, doença do enxerto versus hospedeiro, doença relacionada a IgG4.

\subsection{Tratamento}

\subsubsection{Doença do olho seco}

A etiologia multifatorial, associada à existência de diversos mecanismos, dificulta o manejo da DOS, de modo que é necessário determiná-la (evaporação ou deficiência aquosa), bem como a presença de outras doenças oculares, para selecionar a forma tratamento (Şimşek et al., 2018).

Primeiramente, é necessário orientar o paciente quanto à realização de alterações comportamentais e dietéticas (como a suplementação de ácidos graxos essenciais), possibilidade de suspensão de medicações sistêmicas agravadoras, adição de lubrificantes oculares e higiene palpebral. Caso não haja melhora, deve-se 
optar pela implantação de plugs nos pontos lacrimais, uso de óculos de proteção que mantêm a umidade, aplicação de colírios lubrificantes durante a noite, remoção de obstrução das glândulas de Meibômio por compressas mornas e expressão, e administração de corticoides, antibióticos, secretagogos, ciclosporina e tacrolimus. Em situações graves, se ainda não ocorrer resposta adequada, poderão ser utilizados colírios de soro autólogo, lentes de contato terapêuticas, corticosteroide tópico por longo período, enxerto de membrana amniótica, tarsorrafia e transplante de GS (Jones et al., 2017).

\subsubsection{Síndrome de Sjögren}

Por enquanto não existe cura para os portadores de SS. O tratamento tem por objetivo amenizar sinais e sintomas e melhorar a qualidade de vida dos pacientes, além da modificação no curso da doença para que as sequelas sejam evitadas ou minimizadas. Nos casos de SS secundária, deve-se tratar a enfermidade associada (Felberg; Dantas, 2006).

A xerostomia deve ser avaliada por dentistas e otorrinolaringologistas, sendo possível aliviar sintomas (Furness et al., 2011). Agonistas muscarínicos de uso oral, como a pilocarpina e a cevimelina, agem nos receptores muscarínicos das glândulas estimulando a secreção salivar e lacrimal; lubrificantes oculares, plugs de ponto lacrimal e administração tópica de corticosteroides melhoram sinais e sintomas oculares (Valim et al., 2015); o uso de ciclosporina A tópica retarda a destruição da GL, leva à apoptose dos linfócitos, suprime apoptose das células acinares e da conjuntiva e reduz a infiltração linfo-plasmocitária no tecido glandular (Gunduz; Ozdemir, 1994).

A rituximabe apresenta eficácia na redução de diversas manifestações, como fadiga, acometimento glandular e atividade de doença, além de melhorar parâmetros imunológicos e a qualidade de vida (Devauchelle-Pensec et al., 2014). O uso de hidroxicloroquina não apresenta redução de sintomas como secura e fadiga, mas melhora a dor associada à SS primária (Carsons et al., 2017; Wang et al., 2017). 


\subsection{Justificativa}

As GL e GS apresentam origens semelhantes e possuem tanto tecido epitelial quanto linfoide, o que as torna susceptíveis a um amplo espectro de patologias. Estas, por sua vez, geralmente têm apresentação clínica inespecífica, como edema localizado na topografia glandular, e proptose e desvio ocular no caso da GL. Recentes avanços em exames de imagem, principalmente Tomografia Computadorizada (TC) e RNM têm transformado o diagnóstico e o manejo de doenças da GL e GS, trazendo informações adicionais a respeito dessas características clínicas vagas, como por exemplo a extensão para extruturas vizinhas e a natureza da lesão (Hughes; Miszkiel, 2006).

Em relação à DOS, apesar dos métodos objetivos e quantitativos existentes auxiliarem na detecção de sinais de ressecamento, como inflamação da superfície ocular, dano epitelial e hiperosmolaridade do filme lacrimal, a associação entre os sinais e sintomas da doença é baixa e inconsistente (Batlertt et al., 2015).

A inexistência de consenso para o diagnóstico da DOS se deve, entre outros fatores, à falta de concordância entre seus diferentes conceitos e classificações (Fridman et al., 2004) e à dificuldade em se estabelecer um valor de corte entre olho seco e não seco, uma vez que estes termos não constituem uma dicotomia. Além disso, a influência ambiental contribui para a variabilidade dos resultados dos exames (Savini et al., 2008). A falta de padronização dos testes disponíveis, como ocorre com a presença de diversos tipos de coloração da superfície ocular e seus variados sistemas de graduação, dificulta a comparação entre estudos realizados por diferentes autores (Foulks, 2003). Compararam-se testes diagnósticos existentes (coloração conjuntival e corneana, Schirmer, tempo de quebra do filme lacrimal, osmolaridade da lágrima e questionário sintomático) para diversas condições causadoras de olho seco, e observou-se ampla variação de resultados nos exames realizados, de modo que não foi possível identificar um possível marcador para diagnóstico comum (Alves et al., 2014). A alta sensibilidade ocular a estímulos externos, inclusive aqueles provenientes de exames invasivos, podem influenciar os resultados (Yokoi; Komuro, 2004), assim como, no outro extremo, doenças 
avançadas do olho reduzem sua sensibilidade, enfraquecendo a associação entre sintomas e sinais observados nos testes (Schein et al., 1997; Hay et al., 1998).

Em relação, tanto ao olho seco quanto às queixas de boca seca e à SS, nenhum dos exames disponíveis é suficientemente preditivo para diagnóstico, nem preciso para a correlação com a função glandular lacrimal e salivar. A ausência de padronização para a realização da medida do fluxo salivar e o fato de a biópsia e a sialografia serem exames invasivos contribuem para isso (Hay et al., 1998). O fato de serem adotados diversos critérios diagnósticos propostos por diferentes entidades e sociedades para definir a SS, torna difícil ou quase impossível fazer comparações entre os diversos estudos clínicos, principalmente com relação ao tratamento e aos dados epidemiológicos. Nenhum sinal, achado clínico ou imunomarcador descrito até o momento é aceito isoladamente como ideal para fechar o diagnóstico da SS ou detectar os períodos de atividade e remissão da doença (Felberg; Dantas, 2006).

Diante dos fatos destacados, e considerando o impacto causado pelo acometimento da GL e GP em pacientes com SS, torna-se necessário buscar formas mais eficientes e menos invasivas de avaliação dessas estruturas, visando aprimorar a compreensão da fisiopatogenia da doença e o monitoramento da resposta ao tratamento. O uso cada vez mais difundido de exames de imagem para o diagnóstico e acompanhamento de enfermidades das referidas glândulas motivou o desenvolvimento deste estudo, que pretende correlacionar achados de GL em imagens de RNM com dados clínicos de três grupos: doentes com SS, controles e pacientes com DOS não-SS.

\subsection{Exames de imagem para avaliação de doenças das glândulas lacrimal e parótida}

Exames de imagem como a TC e a RNM permitem a obtenção de diversos parâmetros, como tamanho e densidade dos tecidos estudados, que podem ser medidos e comparados por programas informatizados de análise de imagem, como Osirix (desenvolvido por Pixmeo SARL, Geneva, Suiça) e Clear Canvas (Toronto, Canadá) (Rosset; Spadola; Ratib, 2004; Barra, FR; Barra, RR; Barra, SA, 2010). Isto é possível graças ao padrão tecnológico global utilizado chamado Digital Imaging 
and Communication in Medicine (DICOM), que por sua vez, permite: produção, armazenamento, visualização, processamento, envio e impressão de imagens médicas e documentos correlatos, que podem ser lidos por qualquer programa com capacidade para receber imagens em formato DICOM (Cavalcanti; Sales, 2012).

Entre os potenciais diagnósticos com o uso da TC estão os de alterações de GL, incluindo sua ausência em determinadas síndromes (Duarte et al., 2017). Alterações atróficas e na estrutura interna da GL, mesmo as observadas na senescência, são mais difíceis de serem detectadas na TC e na Ultrassonografia (US) (Ueno et al., 1996).

A RNM, por sua vez, é o método de diagnóstico por imagem não-invasivo mais sensível para avaliar partes moles, particularmente o encéfalo, e possui poucos efeitos deletérios, pois não utiliza radiação. RNM utiliza poderosos ímãs em vez de radiação para formar as imagens. As imagens são produzidas pela interação dos átomos de hidrogênio presentes no corpo com campo magnético de alta energia e pulsos de radiofrequência (RF). Após a aplicação de um campo magnético estático (responsável pelo aparecimento de magnetização), é aplicado outro campo oscilatório, que gira a magnetização em determinado ângulo. Logo após o impulso de RF ter deixado de agir, há tendência natural do sistema de voltar ao estado inicial, devendo ocorrer a reorganização do povoamento do giro dos prótons ao redor do próprio eixo (spin), e a defasagem desse movimento. Esses dois processos correspondem a dois fenômenos de relaxamento: T1 (tempo constante para que a magnetização longitudinal da amostra tecidual retorne ao equilíbrio após a aplicação de um pulso de RF de 90 graus) e T2 (tempo gasto para a magnetização transversal voltar ao estado de menor energia, ou seja, ao vetor de magnetização longitudinal, após aplicação de um pulso de RF de 180 graus). $O$ uso de contraste pode melhorar a visibilidade de determinado tecido, e atua alterando o campo magnético local dos tecidos examinados, que reagem de forma diferente, criando sinais variáveis, permitindo a observação de anormalidades e processos patológicos. O contraste mais utilizado em RNM é o gadolíneo, e seus efeitos colaterais são mínimos, quando comparados com os meios de contraste iodados utilizados na TC (Santos; Cavalcanti, 2012). 
A RNM permite obter dados importantes, como a intensidade de sinal de uma determinada estrutura. Quando o núcleo dos átomos de hidrogênio retorna ao estado de equilíbrio, são emitidos determinados sinais, que por sua vez, são captados pelo computador, construindo uma imagem em preto e branco, a partir da determinação da posição no espaço e da intensidade da energia. Essa intensidade é mostrada como "brilho" na imagem, sendo utilizada a nomenclatura "intensidade de sinal". Estruturas que contenham mais água em sua composição aparecem escuras em T1 e brilhantes em T2, enquanto que tecidos cuja composição apresente gordura aparecem brilhantes em T1 e escuras em T2, o que facilita seu reconhecimento, bem como a constatação de processos patológicos (Cavalcanti, 2012).

A RNM também permite a análise do também do fluxo de íons nos tecidos, a partir da obtenção do chamado coeficiente aparente de difusão (ADC). O ADC, obtido a partir da sequência de difusão $\mathrm{DWI}$, fornece informação biológica a cerca do movimento molecular da água nos meios intra e extracelulares, provocado por mudanças teciduais secundárias a processos patológicos, sendo inversamente proporcional à celularidade do tecido (figura 1). Possui elevadas sensibilidade e especificidade para a diferenciação entre lesões malignas e benignas. Tumores malignos apresentam alta celularidade e, portanto, baixos valores de ADC, pela redução na velocidade com que as moléculas de água se movimentam entre as células, comparativamente às lesões benignas (Elkhamary, 2012).

Figura 1. Deslocamento das moléculas de água entre os espaços intra e extracelular e dentro do espaço extracelular (setas). Em A a movimentação das moléculas de água é restrita devido à maior quantidade de células existentes; em $\mathrm{B}$ a celularidade é menor, com consequente maior movimentação das moléculas.

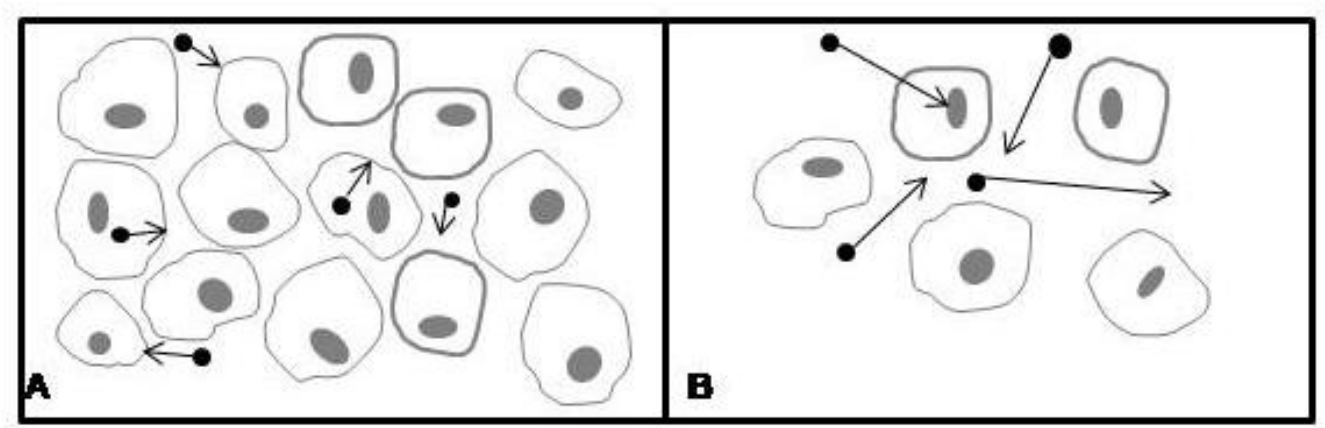


Recentemente, dois casos de olho seco foram elucidados pelo exame de RNM, em que foram observadas GLs ausentes bilateralmente, em crianças com olho seco grave (Sahinoglu et al., 2011; Santo et al., 2013). Em estudo de GS realizado por meio de RNM, os achados contribuíram para o diagnóstico funcional e nosológico da xerostomia, inclusive para detecção precoce de SS (Takagi et al., 2005; Houweling et al., 2011).

A avaliação da GP de portadores de SS à US mostrou que é possível usar esta ferramenta para o diagnóstico de pacientes com a forma primária da doença (Cornec et al., 2013). Trata-se de um exame com baixo custo e amplamente disponível, mas que apresenta a desvantagem de ser operador-dependente e demonstrar menor sensibilidade e especificidade em relação à RNM, que por sua vez, permite obter informações tanto do parênquima quanto dos ductos glandulares (Niemela et al., 2004). Izumi et al. (1996) propuseram um novo critério diagnóstico para SS por meio da análise quantitativa da GP em RNM, em substituição aos métodos invasivos, como biópsia e sialografia.

Devido ao fato de a SS provocar alterações histopatológicas semelhantes na GP e na GL, outro estudo buscou caracterizar imagens da GL à RNM, e as utilizou para explicar os estágios de acometimento durante a progressão da doença (Izumi et al., 1998). 
2. Objetivos 


\subsection{Objetivo geral}

Avaliar se o olho seco está associado a alterações morfológicas da GL à RNM.

\subsection{Objetivos específicos}

1. Avaliar se a GL possui alterações relacionadas ao sexo e à idade no grupo controle;

2. Avaliar se a GL possui alterações relacionadas à SS;

3. Avaliar se os parâmetros clínicos de secura ocular se correlacionam aos achados da RNM nos grupos SS e olho seco não-SS. 
3. Casuística e Métodos 


\subsection{Considerações éticas}

O presente estudo foi aprovado pelo Comitê de Ética em Pesquisa do Hospital das Clínicas da Faculdade de Medicina de Ribeirão Preto da Universidade de São Paulo (HCFMRP-USP), Processo № 1.484 .183 (Anexo A). Todos os pacientes incluídos concordaram em participar no estudo após anuência e assinatura do Termo de Consentimento Livre e Esclarecido (Anexo B).

\subsection{Características da amostra}

\subsection{Etapa 1}

Inicialmente, foram selecionadas imagens de RNM 3.0 tesla do segmento cefálico de 68 pacientes nos arquivos do Serviço de Radiologia do HCFMRP-USP, considerados controles, sendo 40 mulheres e 28 homens de 10 a 89 anos, divididos em quatro grupos etários, a cada 20 anos. As imagens foram avaliadas utilizando-se o processador Clear Canvas (Clear Canvas Inc, Toronto, Canadá), quanto a: volume da GL, em centímetros cúbicos $\left(\mathrm{cm}^{3}\right)$, relação entre a intensidade de sinal da GL e a do vítreo $(\mathrm{GL} / \mathrm{V})$ e o coeficiente aparente de difusão da GL. As médias dos valores encontrados foram comparadas primeiramente entre os grupos e, em seguida, em cada faixa etária, entre os sexos e os olhos direito (OD) e esquerdo (OE).

\subsection{Etapa 2}

Posteriormente, para avaliar as relações dos parâmetros radiológicos da GL com a SS e com a DOS, os pacientes obtidos para o estudo foram aqueles que se encontravam em seguimento no Ambulatório de Oftalmologia do HCFMRP-USP devido à SS ou DOS não-SS. Foram convocados para exame 27 pacientes com SS e 11 com DOS não-SS e, dentre eles, os que não possuíam RNM encefálica realizada foram encaminhados para exame mediante concordância com Termo de Consentimento Esclarecido (Anexo B). Os controles foram selecionados a partir do grupo estudado na primeira etapa, e constituíram-se de 29 indivíduos, que responderam a questionário de três perguntas voltado para sinais e sintomas de 
olho seco, uma vez que houve recusa em comparecer ao exame oftalmológico por parte da maioria deles, além do fato de não ter sido possível sequer o contato telefônico com muitos indivíduos. Os três grupos foram compostos, exclusivamente, por pacientes do sexo feminino e subdivididos em três faixas etárias: 25 a 39 anos, 40 a 59 anos e 60 a 80 anos.

Informações relacionadas a tempo de tratamento para a doença de base e de evolução de outros sinais clínicos e oculares, como olho seco, foram coletadas de prontuários dos casos elegíveis e confirmadas por meio de questionário dirigido sobre os sintomas.

\subsection{Avaliação clínica}

Os pacientes foram avaliados para coleta dos seguintes dados:

1. Anamnese e exame ocular: tempo de tratamento da doença de base, sintomas, acuidade visual corrigida e achados oculares à biomicroscopia.

2. A avaliação da superfície ocular envolveu:

a) questionário dirigido para alterações da superfície ocular (OSDI);

b) teste de Schirmer sem anestésico;

c) Coloração com fluoresceína (1\%) tópica:

i. Quantificação das áreas de irregularidades e desepitelização da córnea, com notas de 0 a 15;

ii. Avaliação do tempo de ruptura do filme lacrimal (TRFL), em segundos.

3. Taxa de fluxo salivar não estimulado (Vitali; Moutsopoulos; Bombardieri, 1994): solicitou-se aos pacientes com SS e olho seco não-SS que expelissem toda a saliva que produzissem durante 15 minutos em um tubo graduado em mililitros, entregue durante a consulta.

O grupo controle foi avaliado por meio de questionário curto de sintomas de DOS reportado no consenso sobre olho seco publicado em 2007 (Dry Eye Workshop Committee, 2007), traduzido para a língua portuguesa e validado previamente 
(Castro et al., 2017). As perguntas, aplicadas via telefonema, em virtude da recusa dos pacientes em comparecerem ao ambulatório do HCFMRP-USP, foram as seguintes:

- Você sente os olhos secos?

- Você sente os olhos irritados?

- Você já teve o diagnóstico de olho seco feito por um médico?

De acordo com a frequência dos sintomas relatados nas duas primeiras perguntas, as respostas obedeciam a um escore de 0 a 3 , que indicavam, respectivamente: nunca, raramente, frequentemente e constantemente. Já a terceira pergunta apresentava apenas as opções "sim" ou "não" como respostas.

\subsection{Avaliação radiológica}

A segunda etapa do estudo incluiu a avaliação da glândula parótida e do gânglio do nervo trigêmeo, pelo fato de tais estruturas poderem ser afetadas pela SS, como exposto anteriormente, e por serem facilmente acessadas à RNM.

As imagens da RNM foram projetadas no Clear Canvas e analisadas, portanto, na segunda etapa do estudo, quanto a:

- Volume da GL;

- Intensidade de sinal da GL/V;

- Intensidade de sinal da GP/V;

- ADC da GL;

- ADC da GP;

- Volume do gânglio trigêmeo (GT)

O volume da GL foi obtido por meio da medição da área da GL em todos os cortes axiais em T1 contrastados, nos quais era possível sua visualização, seguida da soma dos valores encontrados e sua multiplicação pela espessura dos cortes (Figura 2). 
Figura 2 - Imagem de glândula lacrimal normal em corte axial em T1 (setas brancas)

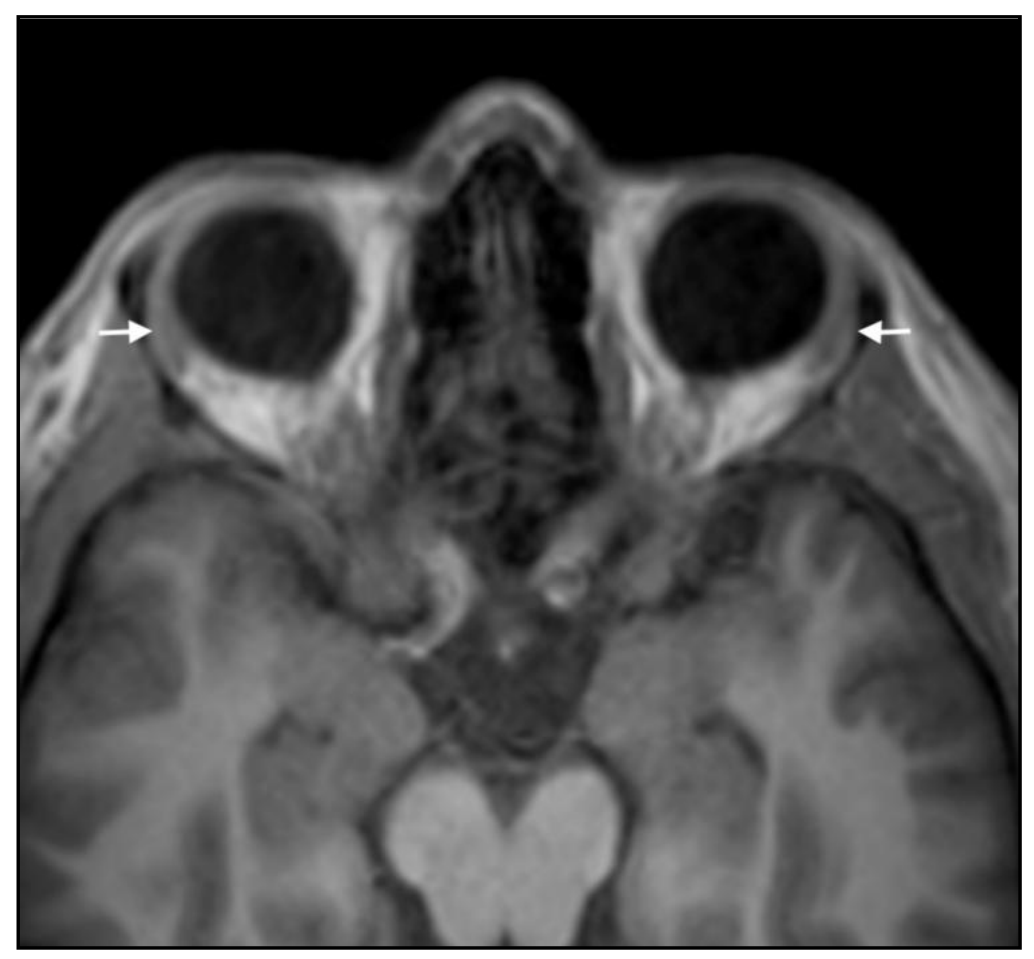

A intensidade relativa de sinal foi analisada em cortes axiais em T2, a partir de fragmentos com área mínima de $0,1 \mathrm{~cm}^{2}$ desenhados na GL, GP (Figura 3) e no vítreo, escolhido para o estudo, por apresentar menor coeficiente de variação em relação a outra estrutura avaliada, o músculo reto medial. 
Figura 3 - Imagem de GP normal em corte axial em T2, com alta intensidade de sinal (asterisco). A assimetria entre a GP direita e a esquerda se deve à rotação no plano transversal

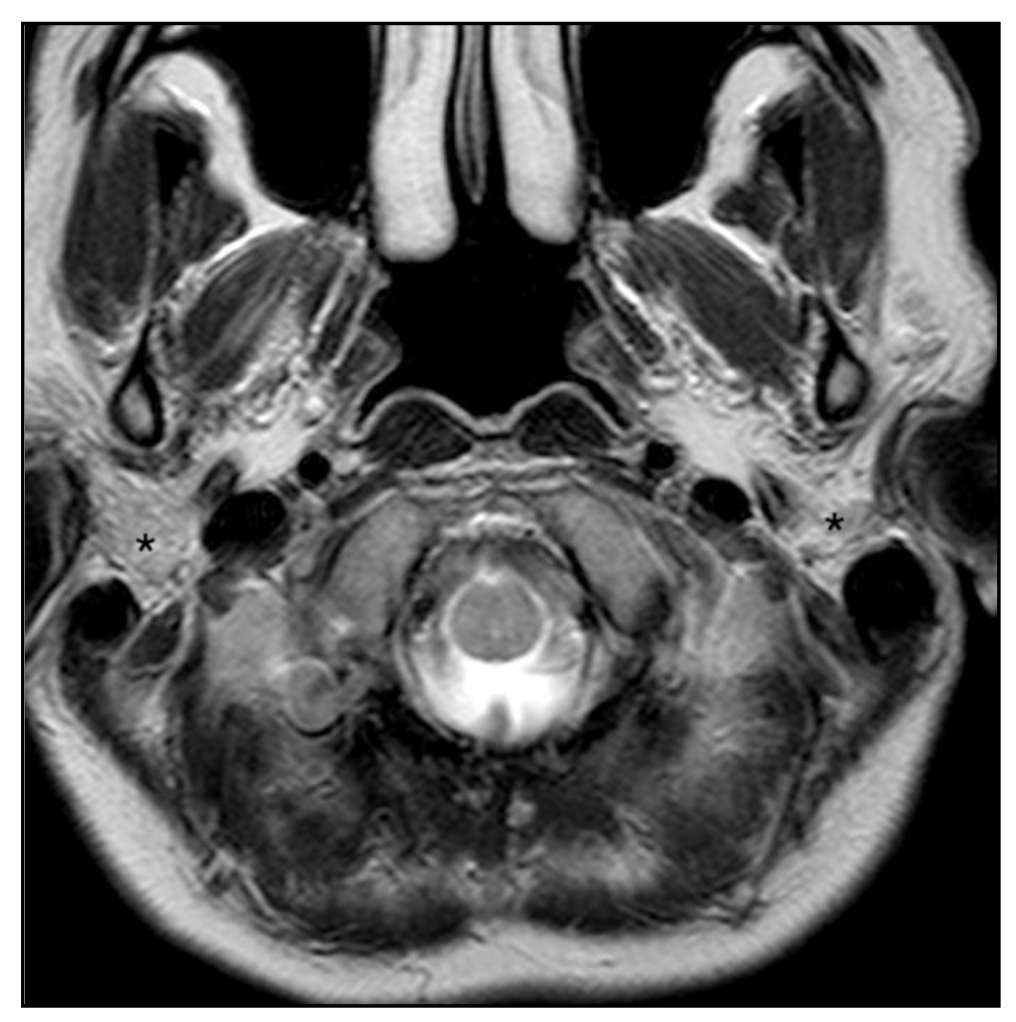

O ADC da GL e da GP foi estudado em sequências de difusão DWI, atribuindo-se valores absolutos em $\mathrm{mm}^{2} / \mathrm{s}$ para a intensidade do sinal de regiões medindo no mínimo $0,1 \mathrm{~cm}^{2}$ das referidas glândulas.

O volume do GT foi obtido da mesma forma que da GL, porém foram utilizados cortes axiais ou coronais em T1 sem contraste do cavum de Meckel (Figura 4). 
Figura 4 - Imagem axial em T1 mostrando a porção cisternal do nervo trigeminal (setas) e o cavum de Meckel (asterisco)

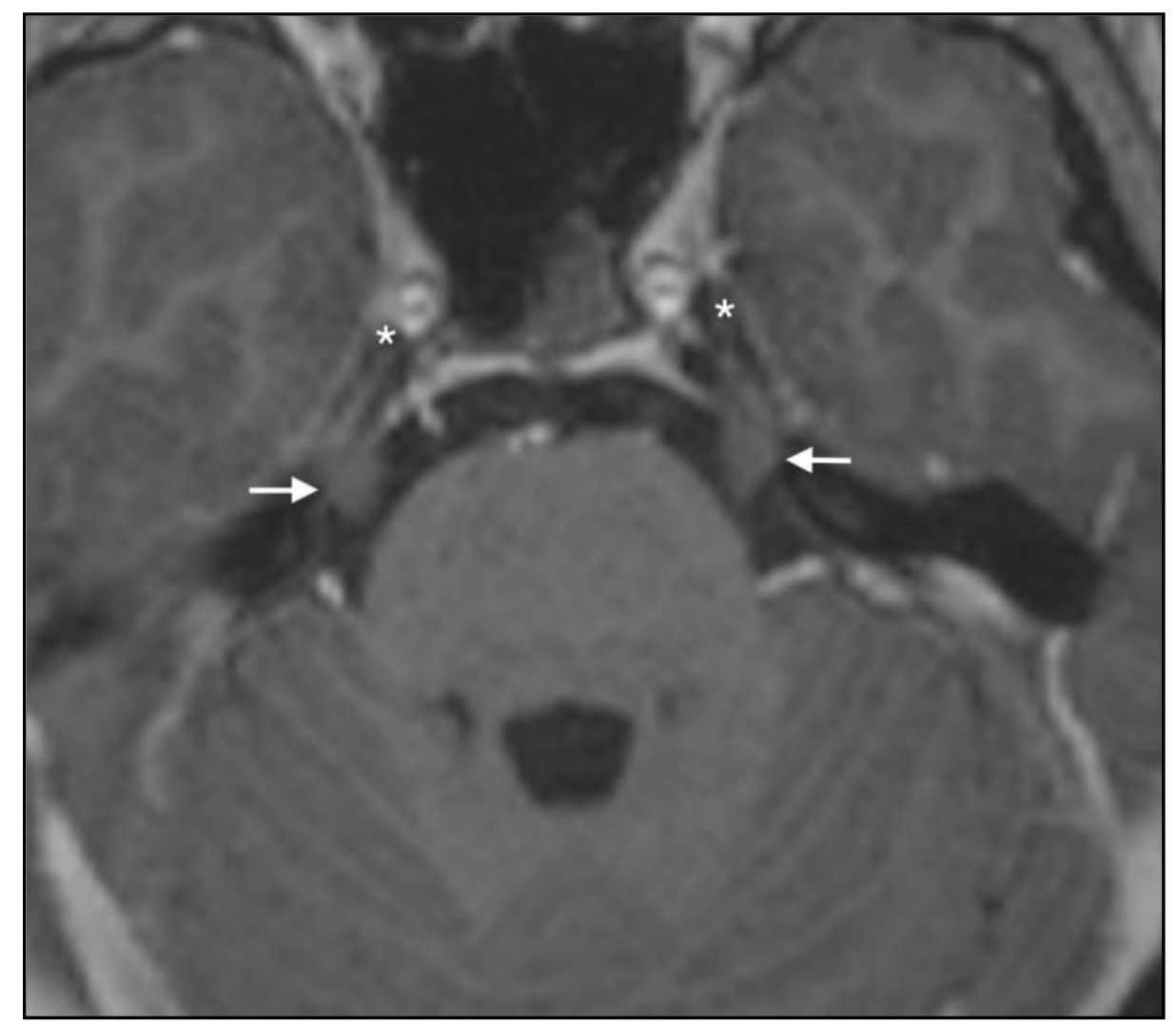

\subsection{Critérios diagnósticos}

O diagnóstico de olho seco foi considerado quando pelos menos um dos seguintes critérios estivesse presente: TRFL $<5$ segundos, teste de Schirmer $<5 \mathrm{~mm}$ em 5 minutos, coloração por fluoresceína >3, ou ainda soma de pontos no questionário de sintomas de irritação ocular $>18$ e a presença de pelo menos 1 queixa de olho seco. O diagnóstico de xerostomia ocorreu quando o resultado do teste do fluxo salivar foi menor do que $0,1 \mathrm{~mL} /$ minuto. Escore igual ou maior que 1 , obtido no questinonário curto e resposta positiva à terceira pergunta foram considerados sugestivos de olho seco. 


\subsection{Critérios de inclusão}

Os pacientes eleitos para este estudo foram aqueles que realizavam seguimento clínico no Ambulatório de Oftalmologia do HCFMRP-USP devido ao diagnóstico de SS ou DOS não-SS, e submetidos ao exame de RNM com indicação relacionada ao seu caso clínico, no qual a GL pôde ser avaliada, e que concordaram em fazer o exame ocular solicitado, no HCFMRP-USP. O diagnóstico de SS foi definido segundo os critérios europeus modificados pelo Grupo de Consenso Americano-Europeu (Vitali et al., 2002). O grupo controle foi consistiuído por indivíduos com idade e sexo similares aos grupos referidos acima, porém sem alterações no exame da GL, e que apresentavam escore menor que 1 ou resposta negativa à terceira pergunta do questionário curto.

\subsection{Critérios de exclusão}

Os pacientes dos grupos doentes cujas informações não puderam ser resgatadas, que não compareceram à convocação, ou não concordaram com os termos do consentimento informado, e também os indivíduos sem alterações no exame da GL com respostas positivas a qualquer uma das três perguntas do questionário curto.

\subsection{Análise estatística}

A análise estatística constou de: estatística descritiva (expressa em médias e desvio padrão - DP); comparações entre os grupos de estudo e entre os grupos etários, das variáveis contínuas (dados radiológicos), pelo teste $\mathrm{F}$ para análise de variância e teste de Tukey para comparação das médias. Posteriormente, foi utilizado o coeficiente de correlação de Pearson para avaliar, entre os doentes, a correlação entre as variáveis clínicas e radiológicas. Em todas as circunstâncias, consideraram-se valores de $\mathrm{p}<0,05$ estatisticamente significativos. 


\subsection{Análise crítica dos riscos e benefícios}

O estudo envolveu métodos propedêuticos não-invasivos ou de toque superficial, leve e rápido na superfície do olho, aplicados na rotina de exames dos pacientes. Desse modo, apenas em poucos casos há risco de produzirem desconforto que, quando presente, persiste por minutos ou poucas horas.

Entre os potenciais benefícios estão os registros sistematizados dos resultados observados, a prática do raciocínio clínico inquisitivo entre a equipe médica acerca dos resultados colhidos rotineiramente, porém avaliados de forma sistematizada, e a possibilidade da identificação de fatores que auxiliem na provável associação entre alterações na imagem da GL à RNM e síndrome do olho seco e seus prováveis mecanismos fisiopatológicos. 
4. Resultados 
Em relação à primeira etapa do estudo, o volume médio da GL diminuiu com o aumento da idade $(p<0,001)$, mas não apresentou diferença estatística entre os sexos e entre OD e OE (Tabela 1). O valor da intensidade relativa de sinal da GL/V foi maior nas faixas etárias de 30 a 49 anos e de 70 a 89 anos (Tabela 2). Observouse coeficiente de difusão da GL do $O E$ maior que do $O D(p=0,003)$, não havendo diferença entre os sexos e entre os grupos etários (Tabela 3).

Tabela 1 - Média do volume da glândula lacrimal do grupo controle

\begin{tabular}{cccc}
\hline Variável estudada & $\mathbf{N}$ & Volume $\mathbf{G L}\left(\mathbf{c m}^{3}\right)$ & $\mathbf{P}$ \\
\hline Faixa etária & 8 & $0,61 \pm 0,20$ & \\
$\mathbf{1 0}$ a $\mathbf{2 9}$ & 25 & $0,42 \pm 0,17$ & \\
$\mathbf{3 0}$ a $\mathbf{4 9}$ & 21 & $0,35 \pm 0,15$ & $<0,001$ \\
$\mathbf{5 0}$ a 69 & 14 & $0,32 \pm 0,14$ & \\
$\mathbf{7 0}$ a 89 & & & \\
Lateralidade & 68 & $0,40 \pm 0,18$ & 0,37 \\
$\quad$ Olho Direito & 67 & $0,37 \pm 0,19$ & \\
Olho Esquerdo & & & 0,85 \\
Sexo & 40 & $0,39 \pm 0,16$ & \\
\hline Feminino & 28 & $0,41 \pm 0,21$ & \\
Masculino & &
\end{tabular}


Tabela 2 - Média da razão intensidade sinal glândula lacrimal/vítreo do grupo controle

\begin{tabular}{cccc}
\hline Variável estudada & $\mathbf{N}$ & Int. sinal GL/V & $\mathbf{P}$ \\
\hline $\begin{array}{c}\text { Faixa etária } \\
\mathbf{1 0} \text { a } \mathbf{2 9}\end{array}$ & 8 & $0,22 \pm 0,04$ & \\
$\mathbf{3 0}$ a 49 & 24 & $0,29 \pm 0,06$ & \\
$\mathbf{5 0}$ a 69 & 21 & $0,25 \pm 0,04$ & 0,003 \\
$\mathbf{7 0}$ a 89 & 14 & $0,30 \pm 0,06$ & \\
Lateralidade & & & \\
Olho Direito & 67 & $0,27 \pm 0,06$ & 0,07 \\
$\quad$ Olho Esquerdo & 67 & $0,29 \pm 0,05$ & \\
Sexo & & & 0,98 \\
Feminino & 39 & $0,27 \pm 0,05$ & \\
Masculino & 28 & $0,26 \pm 0,07$ & \\
\hline
\end{tabular}

Tabela 3 - Média do coeficiente aparente de difusão da glândula lacrimal do grupo controle

\begin{tabular}{cccc}
\hline Variável estudada & $\mathbf{N}$ & Coeficiente de difusão GL & $\mathbf{P}$ \\
\hline Faixa etária & 6 & $1107 \pm 97$ & \\
$\mathbf{1 0}$ a $\mathbf{2 9}$ & 20 & $1071 \pm 128$ & \\
$\mathbf{3 0}$ a $\mathbf{4 9}$ & 18 & $1098 \pm 119$ & 0,60 \\
$\mathbf{5 0}$ a 69 & 14 & $1118 \pm 150$ & \\
$\mathbf{7 0}$ a 89 & & & 0,03 \\
Lateralidade & 58 & $1095 \pm 127$ & \\
Olho Direito & 56 & $1186 \pm 199$ & 0,38 \\
Olho Esquerdo & & & \\
Sexo & 39 & $1125 \pm 149$ & \\
Feminino & 19 & & \\
Masculino & & & \\
\hline
\end{tabular}


Comparando-se os três grupos na segunda etapa do estudo, observou-se que a média do volume do GT foi menor no grupo com SS e maior nos pacientes com olho seco não-SS $(p=0,02)$. O grupo controle demonstrou valores de ADC maiores que os dos outros grupos, embora sem significância estatística (Tabela 4).

Tabela 4 - Comparação entre as médias dos parâmetros da ressonância nuclear magnética

\begin{tabular}{|c|c|c|c|c|}
\hline Variável estudada & Controle (N) & S. Sjögren (N) & Olho seco Não-SS (N) & $\mathbf{P}$ \\
\hline Volume GL $\left(\mathrm{cm}^{3}\right)$ & $0,39 \pm 0,17(29)$ & $0,39 \pm 0,29(27)$ & $0,35 \pm 0,23(11)$ & 0,81 \\
\hline Intensidade de sinal & $0,27 \pm 0,04(28)$ & $0,32 \pm 0,11(27)$ & $0,28 \pm 0,15(10)$ & 0,22 \\
\hline \multicolumn{5}{|l|}{ GL/Vítreo } \\
\hline Intensidade de sinal & $0,37 \pm 0,07(29)$ & $0,45 \pm 0,22(26)$ & $0,48 \pm 0,23(10)$ & 0,07 \\
\hline \multicolumn{5}{|l|}{ GP/Vítreo } \\
\hline $\operatorname{ADC~GL~}\left(\mathrm{cm}^{2} / \mathrm{s}\right)$ & $1078 \pm 101(28)$ & $1006 \pm 400(20)$ & $975 \pm 483(08)$ & 0,73 \\
\hline $\operatorname{ADC} \mathrm{GP}\left(\mathrm{cm}^{2} / \mathrm{s}\right)$ & $958 \pm 134(28)$ & $925 \pm 98(19)$ & $893 \pm 318(09)$ & 0,47 \\
\hline Volume GT $\left(\mathrm{cm}^{3}\right)$ & $2,00 \pm 0,98(19)$ & $1,49 \pm 0,68(27)$ & $2,92 \pm 2,32(10)$ & 0,02 \\
\hline
\end{tabular}

GL= Glândula lacrimal; GP= Glândula parótida; $\mathbf{A D C}=$ Coeficiente aparente de difusão; GT= Glânglio trigêmeo.

Todos os grupos mostraram tendência à redução da média do volume da GL com o aumento da idade, e os pacientes idosos com SS apresentaram os menores valores, apesar de não ter ocorrido diferença estatística (Tabela 5).

Tabela 5 - Média do volume da glândula lacrimal $\left(\mathrm{cm}^{3}\right)$ à ressonância nuclear magnética por faixa etária

\begin{tabular}{ccccc}
\hline Faixa etária (anos) & Controle (N) & S. Sjögren (N) & Olho seco Não-SS (N) & P \\
\cline { 1 - 4 } 25 a $\mathbf{3 9}$ & $\mathbf{P = 0 , 2 2}$ & $\mathbf{P = 0 , 1 2}$ & $\mathbf{P = 0 , 9 7}$ & \\
40 a $\mathbf{5 9}$ & $0,52 \pm 0,25(07)$ & $0,49 \pm 0,30(07)$ & $0,38 \pm 0,38(04)$ & 0,61 \\
$\mathbf{6 0}$ a $\mathbf{8 0}$ & $0,37 \pm 0,12(13)$ & $0,40 \pm 0,32(14)$ & $0,34 \pm 0,26(03)$ & 0,89 \\
\cline { 1 - 4 } & $0,31 \pm 0,11(09)$ & $0,22 \pm 0,10(06)$ & $0,35 \pm 0,20(04)$ & 0,66 \\
\hline
\end{tabular}


As médias da intensidade de sinal da GL/V e da GP/V para cada faixa etária estão descritas nas Tabelas 6 e 7, respectivamente.

Tabela 6 - Média da razão intensidade sinal glândula lacrimal/vítreo à ressonância nuclear magnética por faixa etária

\begin{tabular}{ccccc}
\hline Faixa etária (anos) & Controle (N) & S. Sjögren (N) & Olho seco Não-SS (N) & $\mathbf{P}$ \\
\hline & $\mathbf{P = 0 , 9 1}$ & $\mathbf{P = 0 , 7 1}$ & $\mathbf{P = 0 , 1 4}$ & \\
\hline 25 a $\mathbf{3 9}$ & $0,27 \pm 0,06(07)$ & $0,30 \pm 0,11(07)$ & $0,20 \pm 0,13(04)$ & 0,25 \\
40 a $\mathbf{5 9}$ & $0,28 \pm 0,04(13)$ & $0,31 \pm 0,13(14)$ & $0,30 \pm 0,20(02)$ & 0,71 \\
60 a 80 & $0,26 \pm 0,03(08)$ & $0,35 \pm 0,09(06)$ & $0,34 \pm 0,15(04)$ & 0,23 \\
\hline
\end{tabular}

SS= Síndrome de Jögren.

Tabela 7 - Média da Razão intensidade sinal glândula parótida/vítreo à ressonância nuclear magnética por faixa etária

\begin{tabular}{ccccc}
\hline Faixa etária (anos) & Controle (N) & S. Sjögren (N) & Olho seco Não-SS (N) & $\mathbf{P}$ \\
\hline & $\mathbf{P = 0 , 9 7}$ & $\mathbf{P = 0 , 2 9}$ & $\mathbf{P = 0 , 8 8}$ & \\
\hline $\mathbf{2 5}$ a $\mathbf{3 9}$ & $0,36 \pm 0,05(07)$ & $0,48 \pm 0,23(07)$ & $0,48 \pm 0,16(03)$ & 0,40 \\
$\mathbf{4 0}$ a $\mathbf{5 9}$ & $0,37 \pm 0,08(13)$ & $0,41 \pm 0,19(14)$ & $0,44 \pm 0,05(03)$ & 0,77 \\
$\mathbf{6 0}$ a 80 & $0,38 \pm 0,09(09)$ & $0,55 \pm 0,31(05)$ & $0,41 \pm 0,37(05)$ & 0,19 \\
\hline
\end{tabular}

SS= Síndrome de Jögren

Os coeficientes de difusão da GL e da GP apresentaram médias menores nos pacientes com SS em relação aos controles, embora o valor de $p$ tenha sido maior que 0,05 (Tabelas 8 e 9). 
Tabela 8 - Média do coeficiente de difusão da glândula lacrimal à ressonância nuclear magnética por faixa etária

\begin{tabular}{ccccc}
\hline Faixa etária (anos) & Controle (N) & S. Sjögren (N) & Olho seco Não-SS (N) & $\mathbf{P}$ \\
\hline & $\mathbf{P = 0 , 6 6}$ & $\mathbf{P = 0 , 7 7}$ & $\mathbf{P = 0 , 1 8}$ & \\
\hline 25 a 39 & $986 \pm 54(07)$ & $941 \pm 372(06)$ & $784 \pm 657(04)$ & 0,57 \\
$\mathbf{4 0}$ a $\mathbf{5 9}$ & $1110 \pm 75(12)$ & $1049 \pm 369(11)$ & $1270 \pm 58(02)$ & 0,63 \\
$\mathbf{6 0}$ a $\mathbf{8 0}$ & $1107 \pm 122(09)$ & $978 \pm 683(03)$ & $1061 \pm 63(02)$ & 0,82 \\
\hline
\end{tabular}

Tabela 9 - Média do coeficiente de difusão da glândula parótida à ressonância nuclear magnética por faixa etária

\begin{tabular}{ccccc}
\hline Faixa etária (anos) & Controle (N) & S. Sjögren (N) & Olho seco Não-SS (N) & $\mathbf{P}$ \\
\hline & $\mathbf{P = 0 , 4 2}$ & $\mathbf{P}=\mathbf{0 , 5 1}$ & $\mathbf{P}=\mathbf{0 , 4 4}$ & \\
\hline 25 a 39 & $1021 \pm 155(07)$ & $875 \pm 73(06)$ & $852 \pm 401(04)$ & 0,18 \\
40 a $\mathbf{5 9}$ & $915 \pm 99(12)$ & $963 \pm 89(11)$ & $858 \pm 362(03)$ & 0,58 \\
60 a 80 & $966 \pm 151(09)$ & $866 \pm 175(02)$ & $1029 \pm 133(02)$ & 0,62 \\
\hline
\end{tabular}

Os pacientes idosos portadores de SS demonstraram as menores médias de volume do GT, enquanto que os idosos com olho seco não-SS obtiveram os maiores valores $(p<0,0001)$ (Tabela 10).

Tabela 10 - Média do volume do gânglio trigêmeo $\left(\mathrm{mm}^{3}\right)$ à ressonância nuclear magnética por faixa etária

\begin{tabular}{ccccc}
\hline Faixa etária (anos) & Controle (N) & S. Sjögren (N) & Olho seco Não-SS (N) & $\mathbf{P}$ \\
\hline & $\mathbf{P = 0 , 4 2}$ & $\mathbf{P = 0 , 8 2}$ & $\mathbf{P = 0 , 0 0 1 5}$ & \\
\hline $\mathbf{2 5}$ a $\mathbf{3 9}$ & $2,38 \pm 0,76(06)$ & $1,38 \pm 0,73(07)$ & $2,14 \pm 0,95(04)$ & 0,25 \\
$\mathbf{4 0}$ a 59 & $2,10 \pm 0,64(06)$ & $1,62 \pm 0,66(14)$ & $1,27 \pm 0,02(02)$ & 0,55 \\
$\mathbf{6 0}$ a $\mathbf{8 0}$ & $1,58 \pm 1,13(07)$ & $1,33 \pm 0,72(06)$ & $4,53 \pm 3,02(04)$ & $<\mathbf{0 , 0 0 0 1}$ \\
\hline
\end{tabular}


No grupo com SS, a presença de ceratite puntata, visualizada por meio da coloração corneana por fluoresceína, apresentou correlação positiva $(p=0,03)$ com a intensidade de sinal da GP/V e negativa ( $p=0,0002)$ com o ADC da GL; o volume da GL apresentou médias maiores nos pacientes com maior fluxo salivar $(p=0,01)$, e os doentes com baixa produção de lágrima apresentaram maior intensidade de sinal GP/V (Tabela 11).

Tabela 11 - Correlação entre os parâmetros da ressonância nuclear magnética de pacientes com Síndrome de Sjögren e dados clínicos utilizando o coeficiente de correlação de Pearson

\begin{tabular}{|c|c|c|c|c|c|}
\hline & Fluoresceína & $\overline{\text { TRFL }}$ & Schirmer I & OSDI & Fluxo salivar \\
\hline Volume GL & $-0,24(p=0,21)$ & $-0,02(p=0,91)$ & $0,31(p=0,10)$ & $-0,13(p=0,56)$ & $0,47(p=0,01)$ \\
\hline $\begin{array}{l}\text { Intensidade de } \\
\text { sinal GL/Vítreo }\end{array}$ & $-0,17(p=0,37)$ & $0,07(p=0,69)$ & $-0,23(p=0,23)$ & $0,06(p=0,77)$ & $-0,40(p=0,83)$ \\
\hline $\begin{array}{l}\text { Intensidade de } \\
\text { sinal GP/Vítreo }\end{array}$ & $0,41(p=0,03)$ & $0,14(p=0,46)$ & $-0,44(p=0,02)$ & $-0,20(p=0,29)$ & $0,26(p=0,23)$ \\
\hline $\begin{array}{l}\text { Coeficiente } \\
\text { difusão GL }\end{array}$ & $-0,74(p=0,0002)$ & $0,02(p=0,9)$ & $0,25(p=0,27)$ & $-0,38(p=0,16)$ & $0,37(p=0,10)$ \\
\hline $\begin{array}{l}\text { Coeficiente } \\
\text { difusão GP }\end{array}$ & $0,39(p=0,09)$ & $-0,24(p=0,31)$ & $-0,36(p=0,12)$ & $0,07(p=0,80)$ & $-0,17(p=0,46)$ \\
\hline Volume GT & $0,30(p=0,11)$ & $-0,03(p=0,86)$ & $0,11(p=0,56)$ & $0,03(\mathrm{p}=0,87)$ & $0,26(p=0,17)$ \\
\hline
\end{tabular}

GL= glândual lacrimal; GP= glândula parótida; GT= Gânglio trigêmeo.

A Tabela 12 mostra que não houve correlação entre parâmetros radiológicos e dados clínicos no grupo com olho seco não-SS. 
Tabela 12 - Correlação entre os parâmetros da ressonância nuclear magnética e dados clínicos de pacientes com olho seco não-Síndrome de Sjögren utilizando o coeficiente de correlação de Pearson

\begin{tabular}{lccccc}
\hline & Fluoresceína & TRFL & Schirmer I & OSDI & Fluxo salivar \\
\hline $\begin{array}{l}\text { Volume GL } \\
\begin{array}{l}\text { Intensidade de } \\
\text { sinal GL/Vítreo }\end{array}\end{array}$ & $-0,0,42(p=0,11)$ & $0,08(p=0,76)$ & $-0,09(p=0,74)$ & $-0,33(p=0,25)$ & $0,17(p=0,58)$ \\
$\begin{array}{l}\text { Intensidade de } \\
\text { sinal GP/Vítreo }\end{array}$ & $0,19(p=0,51)$ & $-0,46(p=0,11)$ & $-0,23(p=0,45)$ & $0,35 p=0,28$ & $0,20(p=0,57)$ \\
$\begin{array}{l}\text { Coeficiente } \\
\text { difusão GL }\end{array}$ & $0,25(p=0,51)$ & $0,27(p=0,46)$ & $0,14(p=0,73)$ & $-0,15(p=0,74)$ & $0,55(p=0,19)$ \\
$\begin{array}{l}\text { Coeficiente } \\
\text { difusão GP }\end{array}$ & $0,32(p=0,29)$ & $0,24(p=0,44)$ & $-0,03(p=0,92)$ & $0,14(p=0,68)$ & $0,32(p=0,40)$ \\
\begin{tabular}{l} 
Volume GT \\
\hline
\end{tabular} & $-0,04(p=0,88)$ & $0,29(p=0,30)$ & $0,50(p=0,08)$ & $-0,01(p=0,96)$ & $0,10(p=0,76)$ \\
\hline
\end{tabular}

GL= glândual lacrimal; GP= glândula parótida; GT= Gânglio trigêmeo.

Os dados clínicos de todos os pacientes dos grupos SS e olho seco não-SS podem ser vistos nas tabelas 13 (Anexo C) e 14 (Anexo D), respectivamente. 
Os resultados observados na primeira etapa do presente estudo corroboram os achados descritos em estudo prévio que analisou 260 órbitas sadias por meio de TC, encontrando diminuição do volume da GL com o envelhecimento, sem diferença significativa entre $O$ OD e OE e entre sexo masculino e feminino (Bingham et al., 2013). Outra investigação demonstrou diminuição do tamanho e aumento da intensidade de sinal da GL com o avanço da idade, em exames de RNM de indivíduos sadios (Ueno et al., 1996).

Análises histopatológicas de GL obtidas de autópsias revelaram correlação entre envelhecimento e aumento da incidência de atrofia difusa da porção orbital glandular e fibrose em mulheres, e aumento de fibrose periductal em homens e mulheres, justificando a redução do volume da GL (Obata et al., 1995). Eventos inflamatórios crônicos estariam envolvidos na gênese da atrofia acinar e da fibrose periductal, sendo descoberto que episódios subclínicos repetidos de dacrioadenite ocorrem ao longo da vida na GL, ocasionando obstrução dos ductos secretórios. Tais fatores seriam os responsáveis pela heterogeneidade da GL, e se tornam mais proeminentes com o avanço da idade, justificando aumento na intensidade de sinal (Damato et al., 1984).

A intensidade de sinal observada nas imagens de RNM não é reprodutível por meio de números, sendo apresentada em unidades arbritárias, e seu uso é dependente de alguns fatores (Modic; Herfkens, 1990). Por exemplo, a distância relativa da bobina superficial que capta o sinal eletromagnético afeta a intensidade de sinal do órgão alvo (Ueno et al., 1996). Por esse motivo, nas análises do presente estudo foi utilizada a razão entre as intensidades de sinal das GL e GP e a do corpo vítreo, minimizando possíveis influências externas.

A segunda etapa deste estudo não resultou em diferenças significativas entre entre as GLs dos três grupos analisados. Nos estudos de Fujihara et al. (1999) e Tsubota et al. (2000), pacientes com SS apresentaram GLs aumentadas. Outros autores propuseram um modelo de progressão da SS a partir de dados obtidos de GL examinadas em imagens de RNM. Segundo eles, no estágio inicial da doença a GL apresentaria tamanho normal (medida da área da GL), aparência homogênea e discreto comprometimento funcional (Schirmer próximo do limite inferior), seguidos de hipertrofia da glândula, possivelmente devida à infiltração por células 
inflamatórias, com redução mais acentuada do fluxo lacrimal; posteriormente, a GL voltaria ao tamanho normal, mas demonstrando áreas de alta intensidade de sinal, tornando-se heterogênea e com menores valores ao teste de Schirmer. Nos estágios avançados da síndrome, há atrofia glandular, aumento das áreas com alta intensidade de sinal e comprometimento funcional severo (Izumi et al., 1996). Isso pode justificar os resultados do presente estudo, uma vez que os pacientes analisados possivelmente se encontravam em estágios iniciais da SS, sem comprometimento ainda da estrutura da GL e, consequentemente, de seu tamanho, intensidade de sinal e coeficiente aparente de difusão.

Em realação aos subgrupos etários do grupo controle, na segunda etapa, também não ocorreram diferenças pois foram excluídos os pacientes mais jovens, os quais provavelmente apresentavam glândulas ainda sem processo inflamatório, comprovando a teoria de que em adultos jovens já existe dacrioadenite subclínica, o que justifica o fato de estes últimos não se diferenciarem estatisticamente de idosos sadios.

Comprovou-se que a alta intensidade de sinal da GL na SS era devida à deposição de gordura, já que houve supressão do sinal após aplicação da sequência de recuperação de inversão de tempo curto (STIR). A observação de que a deposição de gordura pode também ocorrer com o envelhecimento em glândulas normais, porém, numa extensão expressivamente menor do que a desenvolvida na SS sugere que seja consequente à destruição da GL devida à infiltração linfocítica e fibrose. Alterações idênticas são notadas, tanto na GL quanto na GP de pacientes portadores de SS (Izumi et al., 1997), justificando o fato de não ter ocorrido diferenças significativas também em relação à GP no presente estudo.

Embora esta investigação não tenha apresentado significância estatística em relação à comparação entre a intensidade média de sinal da GP/V dos três grupos, os pacientes com SS obtiveram os maiores valores. Izumi et al. (1996) determinram que o DP da intensidade de sinal da GP é capaz de distinguir imagens de RNM da GP de pacientes com SS daquelas de indivíduos normais $(p<0.0001)$, sendo que nestes últimos o DP foi menor $(p<0,001)$. Os referidos autores ainda relataram alta correlação entre o padrão da intensidade de sinal e os resultados da biópsia glandular $(r=0,834)$ e da sialografia $(r=0,936)$, na caracterização da SS. 
Sumi et al. (2002) aplicaram a sequência de difusão (DWI) à RNM para avaliar o comprometimento funcional salivar em pacientes com SS, mostrando que o ADC da GP, nesses casos, foi significativamente menor que o de controles e de glândulas inflamadas. Posteriormente, procurou-se estudar a eficácia do ADC em detectar diferenças entre GLs de pacientes com SS e GLs normais. Assim como ocorreu com a GP, pacientes com SS apresentaram GL com menores ADC comprarados com sujeitos sadios. A apoptose das células acinares e a infiltração linfocítica da GL seriam os fatores responsáveis por este achado (Yosuke et al., 2005). Os resultados do presente estudo mostraram menor ADC da GL no grupo SS, e menor ADC da GP nos idosos com SS, embora sem significância estatística, provavelmente em decorrência de não ter havido destruição expressiva do parênquima capaz de levar à diminuição significativa do ADC.

A média do volume do GT apresentou diferença entre os grupos, sendo maior no grupo olho seco não-SS, e menor no grupo SS $(p=0,02)$, em especial nos idosos. Manifestações neurológicas da SS são múltiplas e frequentes, causadas por mecanismos ainda desconhecidos, apesar de serem considerados possíveis fatores desencadeantes a infiltração direta por células monoclonais, presença de anticorpos antineuronais e isquemia secundária à vasculite de pequenos vasos (Tobón et al., 2012). A neuropatia periférica é a mais frequente, presente em 20 a $50 \%$ dos pacientes com SS na forma subclínica, e em 10 a $32 \%$ nos casos manifestados clinicamente (Alexander et. al, 1982; Mellgren et al., 1989; Andonopoulos et. al, 1990). Dentre os tipos de neuropatias periféricas que ocorrem na doença está o envolvimento de nervos cranianos, sendo mais comum o do nervo trigeminal, com predomínio da neuropatia sensorial. A morte neuronal imunomediada no gânglio sensorial de Gasser seria a causa da injúria trigeminal (Mori et al., 2005). No presente estudo, no entanto, ao compararmos as faixas etárias, observou-se que os idosos com olho seco não-SS se diferenciaram dos idosos sadios e dos jovens com olho seco não-SS, não apresentando diferença estatística em relação aos grupos etários com SS. Isso pode ser explicado pelo fato de o número de pacientes do grupo olho seco não-SS ser pequeno, se comparado aos demais, e também por possivelmente se tratarem de achados ao acaso, sem correlação com o quadro de olho seco. 
A análise das correlações entre dados clínicos e os achados radiológicos desta pesquisa mostrou que os pacientes portadores de SS com ceratite puntata (visualizada por meio da coloração corneana por fluoresceína) apresentavam menor $A D C$ da $G L$, indicando que quanto maior o dano estrutural dos tecidos glandulares, maior é o comprometimento funcional, conforme esperado. Também nesse grupo, baixos valores no teste de Schirmer indicam baixa produção de lágrima, e estiveram presentes nos doentes que possuíam maior intensidade de sinal GP/V. Como já citado anteriormente, tanto a GL quanto a GP sofrem as mesmas transformações na SS, porém no momento em que a investigação foi realizada não se observou a mesma correlação com a intensidade de sinal da GL/V, o que poderia se justificar pela possibilidade de a GL ainda se encontrar no estágio de homogeneidade, mas já com redução do fluxo lacrimal.

Por fim, não houve correlação entre os dados radiológicos e clínicos dos pacientes com olho seco não-SS, o que pode ser explicado pelo fato de as mudanças patológicas na GL desses indivíduos serem diferentes das que ocorrem na SS (Damato et al., 1984).

Aplicou-se uma técnica não invasiva para examinar a GL de pacientes com SS, capaz de monitorar alterações intraglandulares características da doença, já que a realização de biópsia pode ser inviável nos casos de atrofia glandular severa.

Este estudo apresentou como fator limitante a análise em um único momento dos pacientes e sem considerar o estágio da doença em que se encontravam, o que pode ter interferido nos resultados, pois conforme demonstrado, a estrutura da GL sofre flutuações de acordo com o estágio de gravidade da SS. Além disso, o baixo número de pacientes do grupo olho seco não-SS e a impossibilidade de se proceder ao exame clínico-oftalmológico do grupo controle podem ter comprometido as análises pretendidas. 
6. Conclusões 
Após análise dos resultados, concluiu-se que:

- A RNM é capaz de detectar alterações na estrutura da GL

- A GL não apresentou alterações relacionadas com o sexo, no grupo controle.

- A GL apresentou alterações relacionadas à idade em indivíduos sadios, sendo seu volume menor com o envelhecimento.

- Não foram encontradas diferenças significativas entre as imagens radiológicas da $\mathrm{GL}$ de pacientes com SS, indivíduos controles e pacientes com olho seco não-SS.

- Pacientes com olho seco não-SS apresentaram alteração neurológica ao exame de RNM.

- No grupo SS, pacientes com baixo fluxo salivar apresentaram menor volume da GL; houve comprometimento da função lacrimal nos casos em que se visualizaram maior intensidade de sinal da GP/V e menor ADC da GL. A menor produção de fluxo lacrimal ocorreu nos pacientes com maior intensidade de sinal da GP/V.

- O grupo com olho seco não-SS não apresentou correlações entre dados clínicos e radiológicos. 


\section{Referências Bibliográficas ${ }^{1}$}

\footnotetext{
${ }^{1}$ Elaboradas de acordo com as Diretrizes para Apresentação de Dissertações e Teses da USP: Documento Eletrônico e Impresso - Parte IV (Vancouver) 3로 ed. São Paulo: SIBi/USP, 2016.
} 
Abe $\mathrm{T}$ et al. Decreased tear lactoferrin concentration in patients with chronic hepatitis C. Br J Ophthalmol. 1999;83(6):684-7.

Alexander EL et al. Neurologic complications of primary Sj̈ogren's syndrome. Medicine. 1982;61(4):247-57.

Alves $\mathrm{M}$ et al. Comparison of diagnostic tests in distinct well-defined conditions related to dry eye disease. PloS One. 2014;9(5): e97921.

American Academy of Ophthalmology Cornea/External Disease Panel. Preferred Practice Pattern Guidelines. Dry Eye Syndrome. San Francisco, CA: American Academy of Ophthalmology, 2013. Disponível em: http://www.aao.org/ppp. Acesso em: 01 jul. 2019.

Andonopoulos AP et al. The spectrum of neurological involvement in Sj'ogren's syndrome. Br J Rheumatol. 1990;29(1):21-3.

Aragona $\mathrm{P}$ et al. Presence of antibodies against Helicobacter pylori and its heatshock protein 60 in the serum of patients with Sjogren's syndrome. J Rheumatol. 1999;26(6):1306-11.

Argüeso ADP et al. Mucin characteristics of human corneal-limbal epithelial cells that exclude the rose bengal. Invest Ophthalmol Vis Sci. 2006; 47(1):113-9.

Arita R, Fukuoka S, Morishige N. New insights into the morphology and function of meibomian glands. Exp Eye Res. 2017;163:64-71.

Asmussen $\mathrm{K}$ et al. Quantitative assessment of clinical disease status in primary Sjogren's syndrome. A cross-sectional study using a new classification model. Scand J Rheumatol. 1997;26(3):197-205.

Asmussen $\mathrm{KH}$, Bowman SJ. Outcome measures in Sjogren's syndrome. Rheumatology (Oxford). 2001;40(10):1085-8. Review.

Barra FR, Barra RR, Barra SA. Visualizadores de imagens médicas gratuitos: é possível trabalhar apenas com eles? Radiol Bras. 2010;43(5):313-8.

Bartlett JD et al. Associations between signs and symptoms of dry eye disease: a systematic review. Clin Ophthalmol. 2015;9:1719-30.

Begley $\mathrm{C}$ et al. Review and analysis of grading scales for ocular surface staining. Ocul Surf. 2019;17(2):208-220.

Bingham CM et al. Calculated CT volumes of lacrimal glands in normal caucasian orbits. Ophthal Plast Reconstr Surg. 2013;29:157-59.

Bron AJ et al. TFOS DEWS II pathophysiology. Ocular Surface, 2017;15(3):438-510. 
Carsons SE et al. Treatment guidelines for rheumatologic manifestations of sjogren's: use of biologics, management of fatigue and inflammatory musculoskeletal pain. Arthritis Care Res. 2017;69(4):517-27.

Castro JS et al. Translation and validation of the Portuguese version of a dry eye disease symptom questionnaire. Arq Bras Oftalmol. 2017;80(1):14-16.

Castro JS et al. Prevalence and Risk Factors of self-reported dry eye in Brazil using a short symptom questionnaire. Sci Rep. 2018;8(1):2076.

Cavalcanti MGP, Sales MAO. Tomografia computadorizada. In: Cavalcanti MGP. Diagnóstico por imagem da face. 2 ${ }^{\mathrm{a}}$ Ed. São Paulo: Livraria Santos Editora Ltda; 2012. p. 3-46.

Cornec D et al. Contribution of salivary gland ultrasonography to the diagnosis of Sjögren's syndrome: toward new diagnostic criteria?Arthritis Rheum. 2013;65(1):21625.

Craig JP et al. TFOS DEWS II report executive summary. Ocul Surf. 2017;15(4):80212.

Credie MG et al. Alterações quantitativas do filme lacrimal após cirurgia refrativa: estudo comparativo entre PRK e LASIK. Arq Bras Oftalmol. 2007;70(1):23-30.

Damato BE et al. Senile atrophy of the human lacrimal gland. The contribution of chronic inflammatory disease. Br J Ophthalmol. 1984;68(9):674-80.

Daniels TE, Whitcher JP. Association of patterns of labial salivary gland inflammation with keratoconjunctivitis sicca. Analysis of 618 patients with suspected Sjogren's syndrome. Arthritis Rheum. 1994;37(6):869-77.

Daniels TE et al. Associations between salivary gland histopathologic diagnoses and phenotypic features of Sjögren's syndrome among 1,726 registry participants. Arthritis Rheum. 2011;63(7):2021-30.

Dantas AM. Anatomia do aparelho visual. Série Oftalmologia Brasileira, Conselho Brasileiro de Oftalmologia. 3a Ed. Rio de Janeiro: Cultura Médica: Guanabara Koogan, 2013. p. 204-5.

Dantas MCN. Olho seco. Arq Bras Oftalmol. 1999;62(1):101-5.

Dartt DA. Neural regulation of lacrimal gland secretory processes: relevance in dry eye diseases. Prog Retin Eye Res. 2009;28(3):155-77.

Davidoff E, Friedman AH. Congenital alacrima. Surv Ophthalmol. 1977;22(2):113-9.

Devauchelle-Pensec $V$ et al. Treatment of primary Sjogren's syndrome with rituximab: a randomized trial. Ann Intern Med. 2014;160(4):233-42. 
Dohlman TH, Ciralsky JB, Lai EC. Tear film assessments for the diagnosis of dry eye. Curr Opin Allergy Clin Immunol. 2016;16(5):487-91.

Doughty MJ et al. A patient questionnaire approach to estimating the prevalence of dry eye symptoms in patients presenting to optometric practices across Canada. Optom Vis Sci. 1997;74:624-31.

Dry Eye Workshop (DEWS) Committee. 2007 Report of the Dry Eye Workshop (DEWS). Ocul Surf. 2007;5(2):65-204.

Duarte AF et al. Lacrimal gland involvement in blepharophimosis-ptosis-epicanthus inversus syndrome. Ophthalmology. 2017;124(3):399-406.

Elkhamary SM. Lacrimal gland lesions: can addition of diffusion-weighted MR imaging improve diagnostic accuracy in characterization? Egypt J Radiol Nucl Med. 2012;43(2):165-72.

Felberg S, Dantas PEC. Diagnóstico e tratamento da síndrome de Sjögren. Arq. Bras. Oftalmol. 2006;69(6):959-63.

Foulks GN. Challenges and pitfalls in clinical trials of treatments for dry eye. Ocul Surf. 2003;1(10:20-30.

Fonseca EC, Arruda GV, Rocha EM. Olho seco: etiopatogenia e tratamento. Arq. Bras. Oftalmol. 2010;73(2):197-203.

Fox RI et al. Sjogren's syndrome. Proposed criteria for classification. Arthritis Rheum. 1986;29(5):577-85.

Fox RI. Sjögren's syndrome. Lancet. 2005;366(9482):321-31.

Fridman $\mathrm{D}$ et al. Olho seco: conceitos, história natural e classificações. Arq Bras Oftalmol. 2004;67:181-5.

Fujihara $T$ et al. Preferential localization of $C D 8+a l p h a E B 7+T$ cells around acinarepithelial cells with apoptosis in patients with Sjögren's syndrome. J Immunol. 1999;163(4):2226-35.

Furness $\mathrm{S}$ et al. Interventions for the management of dry mouth: topical therapies. Cochrane Database Syst Rev. 2011;12:CD008934.

Gayton JL. Etiology, prevalence, and treatment of dry eye disease. Clin Ophthalmol. 2009;3:405-12.

Geerling $G$ et al. Emerging strategies for the diagnosis and treatment of meibomian gland dysfunction: proceedings of the OCEAN group meeting. Ocul Surf. 2017;15(2):179-92. 
Gonzales JA et al. How are ocular signs and symptoms of dry eye associated with depression in women with and without Sjögren's syndrome? Am J Ophthalmol. 2018;191:42-8.

Gunduz K, Ozdemir O. Topical cyclosporin treatment of keratoconjunctivitis sicca in secondary Sjogren's syndrome. Acta Ophthalmol (Copenh). 1994;72(4):438-42.

Hay EM et al. Weak association between subjective symptoms or and objective testing for dry eyes and dry mouth: results from a population based study. Ann Rheum Dis. 1998;57(1):20-4.

Hayashi Y, Arakaki R, Ishimaru N. Apoptosis and estrogen deficiency in primary Sjogren syndrome. Curr Opin Rheumatol. 2004;16(5):522-6.

Holland EJ, Mannis MJ, Lee W.B. Doenças da superfície ocular: córnea, conjuntiva e filme lacrimal. Rio de Janeiro: Elsevier Editora; 1998. p. 39.

Houweling $\mathrm{AC}$ et al. MRI to quantify early radiation-induced changes in the salivary glands. Radiother Oncol. 2011;100(3):386-9.

Hughes GK, Miszkiel KA. Imaging of the lacrimal gland. Semin Ultrasound CT MR. $2006 ; 27(6): 476-91$.

Ivanir $\mathrm{Y}$ et al. Prevalence of dry eye syndrome after allogeneic hematopoietic stem cell transplantation. Cornea. 2013;32(5):e97-e101.

Izumi M et al. MR imaging of the parotid gland in Sjögren's syndrome: A proposal for new diagnostic criteria. AJR Am J Roentgenol. 1996;166(6):1483-7.

Izumi $\mathrm{M}$ et al. Premature fat deposition in the salivary glands associated with Sjögren syndrome: MR and CT evidence. Am J Neuroradiol. 1997;18(5):951-8.

Izumi M et al. MR features of the lacrimal gland in Sjögren's syndrome. AJR Am J Roentgenol. 1998;170(6):1661-6.

Javadi MA, Feizi S. Dry eye syndrome. J Ophthalmic Vis Res. 2011;6(3):192-8.

Jones $\mathrm{L}$ et al. TFOS DEWS II management and therapy report. Ocul Surf. 2017;15(3):575-628.

Kassan SS. Immunogenetics of Sjögren's syndrome. Ann Med Interne $1998 ; 149(1): 45-8$.

Kruszka P, O'Brian RJ. Diagnosis and management of Sjögren's syndrome. Am Fam Physician. 2009;79(6):465-70.

Lee AJ et al. Prevalence and risk factors associated with dry eye symptoms: a population based study in Indonesia. Br J Ophthalmol. 2002;86(12):1347-51. 
Lemp MA. Report of the National Eye Institute / Industry Workshop on Clinical Trials in Dry Eyes. CLAO J. 1995;21(4):221-32.

Lin PY et al. Prevalence of dry eye among an elderly Chinese population in Taiwan: The Shihpai eye study. Ophthalmology. 2003;110(6):1096-101.

Llorens-Quintana C et al. A novel automated approach for infrared-based assessment of meibomian gland morphology. Trans Vis Sci Tech. 2019;8(4):17.

Masmali AM, Purslow C, Murphy PJ. The tear ferning test: a simple clinical technique to evaluate the ocular tear film. Clin Exp Optom. 2014;97(5):399-406.

Murube $\mathrm{J}$ et al. The triple classification of dry eye for practical clinical use. Eur $\mathrm{J}$ Ophthalmol. 2005;15(6):660-7.

McCarty CA et al. The epidemiology of dry eye in Melbourne, Australia. Ophthalmology. 1998;105(6):1114-9.

Mellgren SI et al. Peripheral neuropathy in primary Sj̈ogren's syndrome. Neurology, 1989;39(3):390-4.

Messmer EM. The pathophysiology, diagnosis and treatment of dry eye disease. Dtsch Arztebl Int. 2015;112(5):71-82.

Modic MT, Herfkens RJ. Intervertebral disc. Normal age-related changes in MR signal intensity. Radiology. 1990;177(2):332-3; discussion 333-4.

Módulo CM et al. Influence of insulin treatment on the lacrimal gland and ocular surface of diabetic rats. Endocrine. 2009;36(1):161-8.

Mori $\mathrm{K}$ et al. The wide spectrum of clinical manifestations in Sj̈ogren's syndromeassociated neuropathy. Brain. 2005;128(11):2518-34.

Moutsopoulos HM. Sjögren's syndrome: autoimmune epithelitis. Clin Immunol Immunopathol. 1994;72(2):162-5.

Navazesh M. Methods for collecting saliva. Ann N Y Acad Sci. 1993;694:72-7.

Niemela RK et al. Ultrasonography of salivary glands in primary Sjogren's syndrome: a comparison with magnetic resonance imaging and magnetic resonance sialography of parotid glands. Rheumatology (Oxford). 2004;43(7):875-9.

Obata $\mathrm{H}$ et al. Histopathologic study of human lacrimal gland. Statistical analysis with special reference to aging. Ophthalmology. 1995;102(4):678-86.

Qin B et al. Epidemiology of primary Sjogren's syndrome: a systematic review and meta-analysis. Ann Rheum Dis. 2015;74(11):1983-9. 
Rosset A, Spadola L, Ratib O. OsiriX: an open-source software for navigating in multidimensional DICOM images. J Digit Imaging. 2004;17(3):205-16.

Sahinoglu $\mathrm{N}$ et al. Isolated form of congenital bilateral lacrimal gland agenesis. Indian J Ophthalmol. 2011;59(6):522-3.

Santo RO et al. Giant dacryocystocele and congenital alacrimia in lacrimo-auriculodento-digital syndrome. Ophthalmic Plast Reconstr Surg. 2013;29(3):e67-8.

Santos DT, Cavalcanti MGP. Ressonância magnética. In: Cavalcanti MGP. Diagnóstico por imagem da face. $2^{-}$Ed. São Paulo: Livraria Santos Editora Ltda; 2012. p. 47-58.

Savini G et al. The challenge of dry eye diagnosis. Clin Ophthalmol. 2008;2(1):31-55.

Schaumberg DA et al. Hormone replacement therapy and dry eye syndrome. JAMA. $2001 ; 286(17): 2114-9$.

Schaumberg DA et al. Prevalence of dry eye syndrome among US women. Am J Ophthalmol. 2003;136(2):318-26.

Schein OD et al. Relation between signs and symptoms of dry eye in the elderly: a population-based perspective. Ophthalmology. 1997;104(9):1395-401.

Shiboski $\mathrm{CH}$ et al. 2016 American College of Rheumatology/European League Against Rheumatism classification criteria for primary Sjögren's syndrome: a consensus and data-driven methodology involving three international patient cohorts. Ann Rheum Dis. 2017;76(1):9-16.

Shimmura S, Shimazaki J, Tsubota K. Results of a population-based questionnaire on the symptoms and lifestyles associated with dry eye. Cornea. 1999;18(4):408-11.

Şimşek $\mathrm{C}$ et al. Current management and treatment of dry eye disease. Turk $\mathrm{J}$ Ophthalmol 2018;48(6):309-13.

Stapleton F et al. TFOS DEWS II epidemiology report. Ocul Surf. 2017;15(3),334-65.

Stefanski $\mathrm{AL}$ et al. The Diagnosis and treatment of Sjögren's syndrome. Dtsch Arztebl Int. 2017;114(20):354-61.

Stern ME et al. The role of the lacrimal functional unit in the pathophysiology of dry eye. Exp Eye Res. 2004;78(3):409-16. Review.

Sullivan DA et al. TFOS DEWS II sex, gender, and hormones report. Ocul Surf. 2017;15(3):284-333.

Sumi M et al. Diffusionweighted echoplanar MR imaging of the salivary glands. AJR Am J Roentgenol. 2002;178(4):959-65. 
Takagi $Y$ et al. MR microscopy of the parotid glands in patients with Sjogren's syndrome: quantitative MR diagnostic criteria. AJNR Am $J$ Neuroradiol. 2005;26(5):1207-14.

Tei $M$ et al. Vitamin A deficiency alters the expression of mucin genes by the rat ocular surface epithelium. Invest Ophthalmol Vis Sci. 2000;41(1):82-8.

Terry MA. Dry eye in the elderly. Drugs Aging. 2001;18(2):101-7.

Tobón GJ et al. Neurological disorders in primary Sjogren's syndrome. Autoimmune Dis. 2012;2012:645967.

Tomlinson A et al. The international workshop on meibomian gland dysfunction: report of the diagnosis subcommittee. Invest Ophthalmol Vis Sci. 2011;52(4):200649.

Tong L, Koh V, Thong BY. Review of autoantigens in Sjögren's syndrome: an update. J Inflamm Res. 2017;10:97-105.

Tsubota $\mathrm{K}$ et al. Lacrimal gland function and lymphocytes infiltration in Mikulicz's disease and Sjögren's syndrome. Invest Ophthalmol Vis Sci. 2000;41(7):1666-73.

Ueno $\mathrm{H}$ et al. MR imaging of the lacrimal gland. Age-related and gender-dependent changes in size and structure. Acta Radio. 1996:37(5):714-9.

Valim V et al. Recommendations for the treatment of Sjögren's syndrome. Rev Bras Reumatol. 2015;55(5):446-57.

van Bijsterveld OP. Diagnostic tests in the Sicca syndrome. Arch Ophthalmol. $1969 ; 82(1): 10-4$.

Vitali C, Moutsopoulos HM, Bombardieri S. The European Community Study Group on Diagnostic Criteria for Sjögren's Syndrome. Sensitivity and specificity of tests for ocular and oral involvement in Sjögren's Syndrome. Ann Rheum Dis. 1994;53(10):637-47.

Vitali $\mathrm{C}$ et al. Classification criteria for Sjogren's syndrome: a revised version of the European criteria proposed by the American-European Consensus Group. Ann Rheum Dis. 2002;61(6):554-8. Review.

Volpini $M$ et al. Série Oftalmologia Brasileira. Órbita, Sistema Lacrimal e Oculoplástica. 3를 Ed. Rio de Janeiro: Cultura Médica, Guanabara Koogan, 2013. p. 137-40.

Wakamatsu TH, Dogru M, Tsubota K. Tearful relations: oxidative stress, inflammation and eye diseases. Arq Bras Oftalmol. 2008;71(6 Suppl):72-9. 
Wang $S Q$ et al. Is hydroxychloroquine effective in treating primary Sjogren's syndrome: a systematic review and meta-analysis. BMC Musculoskelet Disord. 2017;18(1):186.

Whitcher JP et al. A simplified quantitative method for assessing keratoconjunctivitis sicca from the Sjogren's Syndrome International Registry. Am J Ophthalmol. 2010;149(3):405-15.

Wolffsohn JS et al. TFOS DEWS II diagnostic methodology report. Ocul Surf. 2017;15(3):539-74.

Yamamoto K. Pathogenesis of Sjogren's syndrome. Autoimmun Rev. 2003;2(1):13-8.

Yokoi N, Komuro A. Non-invasive methods of assessing the tear film. Experiment Eye Res. 2004;78(3):399-407.

Yosuke $\mathrm{K}$ et al. Diffusion-weighted MR microimaging of the lacrimal glands in patients with Sjögren's syndrome. Am J Roentgenol. 2005;184(4):1320-5.

Yu J, Asche CV, Fairchild CJ. The economic burden of dry eye disease in the United States: a decision tree analysis. Cornea. 2011;30(4):379-87.

Zeev MS, Miller DD, Latkany R. Diagnosis of dry eye disease and emerging technologies. Clin Ophthalmol. 2014;8:581-90. 
8. Anexos 


\section{ANEXO A - COMPROVANTE DE APROVAÇÃo do COMITÊ DE ÉTICA}

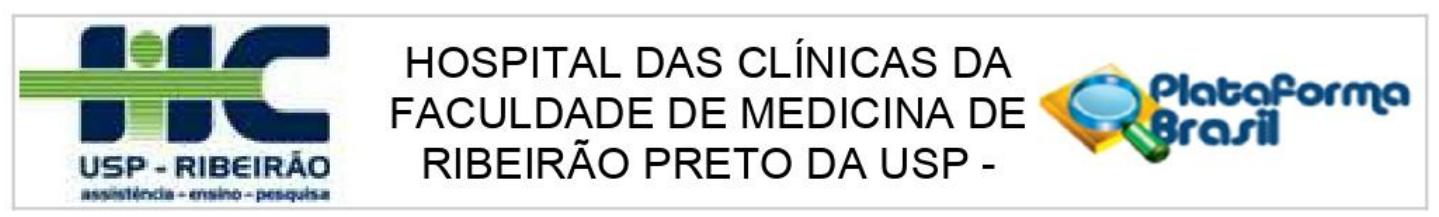

\section{PARECER CONSUBSTANCIADO DO CEP}

\section{DADOS DO PROJETO DE PESQUISA}

Título da Pesquisa: Diagnóstico por Imagem na detecção de alterações da glândula lacrimal com impacto funcional

Pesquisador: Eduardo Melani Rocha

Área Temática:

Versão: 3

CAAE: 12557913.7 .0000 .5440

Instituição Proponente:Hospital das Clínicas da Faculdade de Medicina de Ribeirão Preto da USP -

Patrocinador Principal: Financiamento Próprio

\section{DADOS DA NOTIFICAÇÃO}

Tipo de Notificação: Outros

Detalhe: Troca de pesquisador principal

Justificativa: A função de pesquisador principal foi alterada pela saida do Dr. Marcelo Caram

Data do Envio: 29/03/2016

Situação da Notificação: Parecer Consubstanciado Emitido

\section{DADOS DO PARECER}

Número do Parecer: 1.484 .183

Apresentação da Notificação:

Tipo de Notificação: Outros

Detalhe: Troca de pesquisador principal

Justificativa: A função de pesquisador principal foi alterada pela saida do Dr. Marcelo Caram Ribeiro Fernandes dessa atividade. A função será exercida pelo Prof. Dr. Eduardo Melani Rocha. O pedido de fazer a mudança na folha de rosto do projeto não pde ser feita no sistema, como solicitado pelo CEP.

Data do Envio: 29/03/2016

Objetivo da Notificação:

Trata-se de notificação encaminhando a solicitação de mudança de pesquisador responsável

Endereço: CAMPUS UNIVERSITÁRIO

Bairro: MONTE ALEGRE CEP: $14.048-900$

UF: SP Municipio: RIBEIRAO PRETO

Telefone: (16)3602-2228 Fax: (16)3633-1144 E-mail: cep@hcrp.usp.br 


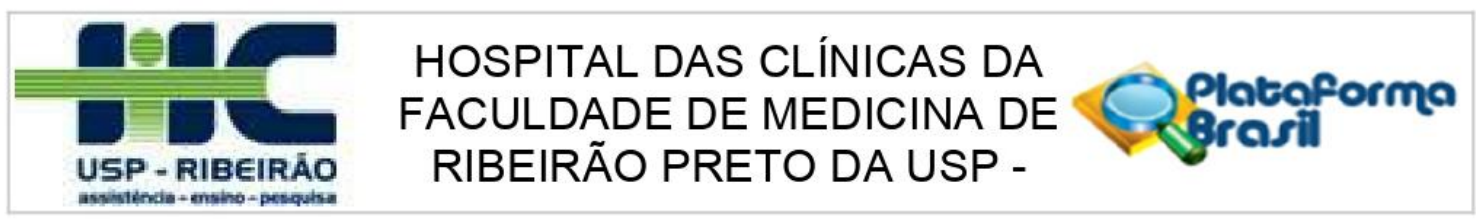

Continuação do Parecer: 1.484.183

conforme carta datada de 28/03/2016 referente à pesquisa supracitada.

Avaliação dos Riscos e Benefícios:

Não se aplica.

Comentários e Considerações sobre a Notificação:

O pesquisador responsável encaminhou para apreciação do CEP a solicitação de mudança de pesquisador responsável do Dr. Marcelo Caram Ribeiro Fernandes para Prof. Dr. Eduardo Melani Rocha, conforme carta datada de 28/03/2016 referente à pesquisa supracitada.

Considerações sobre os Termos de apresentação obrigatória:

Todos os documentos referentes à notificação da pesquisa supracitada foram apresentados ao CEP.

\section{Recomendações:}

Conclusões ou Pendências e Lista de Inadequações:

O CEP tomou ciência da notificação de mudança de pesquisador responsável do Dr. Marcelo Caram Ribeiro Fernandes para Prof. Dr. Eduardo Melani Rocha, conforme carta datada de 28/03/2016 referente à pesquisa supracitada.

Considerações Finais a critério do CEP:

Este parecer foi elaborado baseado nos documentos abaixo relacionados:

\begin{tabular}{|l|l|c|l|r}
\hline \multicolumn{1}{|c|}{ Tipo Documento } & Arquivo & Postagem & Autor & Situaçã \\
\hline Outros & CartaCEPPrjlmagem.pdf & $\begin{array}{c}29 / 03 / 2016 \\
13: 53: 47\end{array}$ & $\begin{array}{l}\text { Marcelo Caram } \\
\text { Ribeiro Fernandes }\end{array}$ & Aceitc \\
\hline
\end{tabular}

Situação do Parecer:

Aprovado

Necessita Apreciação da CONEP:

Não

RIBEIRAO PRETO, 31 de Março de 2016

Assinado por:
MARCIA GUIMARÃES VILLANOVA
(Coordenador)

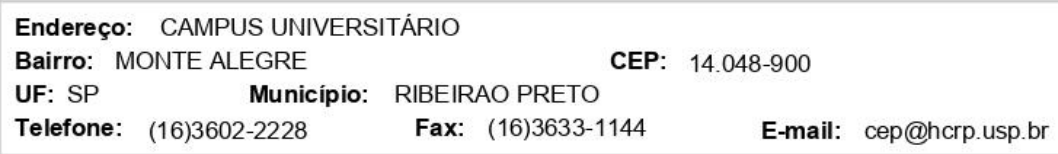




\section{ANEXO B - TERMO DE CONSENTIMENTO LIVRE E ESCLARECIDO}

\section{A. Projeto: Correlação entre parâmetros clínicos e de Ressonância Nuclear Magnética da Síndrome de Sjögren e de pacientes com olho seco não-Sjögren.}

B. Responsáveis pela condução das pesquisas: Dr. Eduardo Melani Rocha, Dra Amanda Pires Barbosa.

C. Nome do paciente:

$\mathrm{HC}$ :

Idade

Sexo: $R G:$

Endereço:

D. Responsável legal:

Grau de parentesco:

Idade:

$R G:$

Endereço:

Forneceu autorização? Sim

Não

E. O trabalho tem como objetivo avaliar a presença de alteração nos olhos e na lágrima de pacientes com exame sugestivo de alteração da glândula lacrimal no exame de ressonância nuclear magnética.

F. O trabalho será realizado com pacientes que se submeteram ao exame de RNM no HCRP-FMRP-USP.

G. A avaliação dos olhos incluirá questionário com perguntas sobre alterações nos olhos, exame ocular, teste de Schirmer com anestésico (medida da produção de lágrima com fitas de papel), exame das pálpebras, avaliação do tempo de ruptura da lágrima, teste com corante Rosa bengala, e coleta de uma gota de lágrima para dosagem de enzimas pelo método ELISA (exame de laboratório) e coleta de células da superfície da conjuntiva com uma fita de papel. Será feito registro das medicações em uso.

H. O paciente deverá comparecer ao Hospital de Clínicas da FMRP-USP no ambulatório de Oftalmologia para avaliação única para cumprir os exames desse estudo. A lágrima será coletada através de pipeta plástica estéril para posterior avaliação em nível laboratorial. Na ocasião o paciente deverá responder ao questionário, e será realizado exame ocular já descrito anteriormente.

I. O paciente será beneficiado na medida em que o esclarecimento da intensidade e frequência de alterações oculares relacionadas ao olho seco permitirá estimar efeitos colaterais não previsíveis no exame de rotina, com repercussões potenciais a longo prazo. 
J. Em relação aos possíveis transtornos: os colírios com corante podem, eventualmente, escorrer pelo rosto do paciente, mas são facilmente removíveis com água corrente. $\mathrm{O}$ teste de Schirmer é indolor e consiste em pingar colírio anestésico em ambos os olhos e, minutos após, colocar uma fita de papel de filtro entre o globo ocular e a pálpebra inferior que absorverá a lágrima; após 5 minutos será medida a absorção de lágrima na fita com uma régua milimetrada. A coleta de lágrimas e células pode causar desconforto, mas este pode ser atenuado com colírio anestésico.

K. As dúvidas sobre quaisquer procedimentos ou outros dados da pesquisa serão esclarecidas através de contato telefônico ou visita ao ambulatório de oftalmologia.

L. O paciente poderá deixar de participar da pesquisa em qualquer momento, sem prejuízo no seu atendimento rotineiro.

M. Será mantida a privacidade do paciente e o caráter confidencial das informações, ou seja, os resultados com a identificação do paciente só serão do conhecimento deste e da equipe médica que o atende. A identidade do paciente será mantida em confidencial e não será, sob qualquer hipótese, exposta nas conclusões ou publicações posteriores.

N. Será disponibilizado tratamento médico em caso de danos diretamente causados pela pesquisa.

O. As informações obtidas durante o estudo serão repassadas a ele ou ela, mesmo que possam vir a afetar a vontade do paciente de continuar no trabalho.

P. Para qualquer dúvida ou informação:

Dr. Eduardo Melani Rocha

Dra Amanda Pires Barbosa

Oftalmologia - (16) 3602-2523, emrocha@fmrp.usp.br

Hospital de Clínicas da FMRP-USP

Ribeirão Preto, / / /2015

Paciente ou representante legal.

Médico responsável: 


\section{Anexo C -}

Tabela 13 - Dados clínicos dos pacientes do grupo Síndrome de Sjögren

PACIENTE IDADE SCHIRMER FLUORESCEÍNA TRFL FLUXO SALIVAR OSDI

$$
(\mathrm{mm})
$$

0774405K $54 \quad 10$

0461755D $70 \quad 4$

0816371F $52 \quad 7$

0080115F $44 \quad 25$

0506946B $52 \quad 17$

0010310 $49 \quad 0$

1303255E $39 \quad 8$

0400297G $31 \quad 28$

0809123G $55 \quad 2$

1184390J $38 \quad 2$

0356579A $72 \quad 7$

1224462। $32 \quad 4$

0270780B $70 \quad 5$

0126437G $49 \quad 30$

0803010C $36 \quad 0$

119358I $39 \quad 22$

1203504E $39 \quad 8$

1360957I $45 \quad 20$

1402548B $59 \quad 14$

0218510E $49 \quad 30$

0456524C $65 \quad 0$

0156727B $53 \quad 30$

0167366G $53 \quad 7$

1404415H $49 \quad 0$

0305883E $49 \quad 3$

1461932H $55 \quad 5$

1132833A $63 \quad 3$ (s) $(\mathrm{mL} / \mathrm{min})$

$2 \quad 0,2 \quad 63$

$3 \quad 0,06 \quad 61,36$

$3 \quad 0,13 \quad 34,09$

$>10 \quad 0,13 \quad 63$

$3 \quad 0,06 \quad 66,66$

$2 \quad 0,3 \quad 66,66$

$>10 \quad 0,13 \quad 12,5$

$20,1 \quad 52$

$4 \quad 0,13 \quad 66,66$

$20,1 \quad 52$

$1 \quad 0,23 \quad 45$

$30 \quad 83,33$

$6 \quad 0,06 \quad 50$

$2 \quad 0,1 \quad 70,45$

$5 \quad 0,25$

$4 \quad 0,4$

$7 \quad 0,04$

77,27

$0 \quad 0,11$

$0 \quad 0,09$

$3 \quad 0,27$

30

6,81

59,09

82,5

10

93,18

$3 \quad 0,16$

79,54 


\section{Anexo D -}

Tabela 14 - Dados clínicos dos pacientes do grupo olho seco Não-Sjögren

PACIENTE IDADE SCHIRMER FLUORESCEÍNA TRFL FLUXO SALIVAR OSDI
$(\mathrm{mm})$
(s) $\quad(\mathrm{mL} / \mathrm{MIN})$
(\%)

$\begin{array}{ccccccc}\text { 1177222B } & 49 & 0 & 6 & 1 & 0,16 & 77,08 \\ \text { 1457148G } & 26 & & 3 & 2 & & 37,5 \\ \text { 1416560K } & 55 & 7 & 0 & 2 & 0,33 & 70,45 \\ \text { 1258667B } & 24 & 0 & 3 & 1 & 0,13 & 81,81 \\ \text { 1036171F } & 56 & 5 & 0 & 5 & 0 & 0 \\ \text { 0177728F } & 67 & 7 & 0 & 7 & 0,2 & 36,36 \\ \text { 0003110K } & 61 & 5 & 0 & 3 & 0 & 60,41 \\ \text { 0098270I } & 36 & 0 & 12 & 3 & 0,4 & 35,41 \\ \text { 0054907F } & 66 & 25 & 0 & 5 & 3 & 0,06 \\ \text { 1155338K } & 36 & 5 & 0 & 3 & 0,02 & 68,18\end{array}$

